\title{
Deep Multimodal Learning: Merging Sensory Data for Massive MIMO Channel Prediction
}

\author{
Yuwen Yang, Feifei Gao, Chengwen Xing, Jianping An, and Ahmed Alkhateeb
}

\begin{abstract}
Existing work in intelligent communications has recently made preliminary attempts to utilize multi-source sensing information (MSI) to improve the system performance. However, the research on MSI aided intelligent communications has not yet explored how to integrate and fuse the multimodal sensory data, which motivates us to develop a systematic framework for wireless communications based on deep multimodal learning (DML). In this paper, we first present complete descriptions and heuristic understandings on the framework of DML based wireless communications, where core design choices are analyzed in the view of communications. Then, we develop several DML based architectures for channel prediction in massive multipleinput multiple-output (MIMO) systems that leverage various modality combinations and fusion levels. The case study of massive MIMO channel prediction offers an important example that can be followed in developing other DML based communication technologies. Simulation results demonstrate that the proposed DML framework can effectively exploit the constructive and complementary information of multimodal sensory data to assist the current wireless communications.
\end{abstract}

Index Terms-Deep multimodal learning (DML), deep learning, wireless communications, channel prediction, massive MIMO

\section{INTRODUCTION}

To satisfy the demand of explosive wireless applications, e.g., diverse intelligent terminal access, autonomous driving, and Internet of Things, etc, the new generation of wireless communication systems is expected to handle massive data and meet the requirements of both high-reliability and low-latency. However, existing communication systems, which are basically designed based on conventional communication theories, exhibit several inherent limitations in meeting the aforementioned requirements, such as relying on accurate theoretical models, suffering from high complexity algorithms, and being restricted to block-structure communication protocols, etc [1][3]. Recently, intelligence communication has been recognized as a promising direction in future wireless communications. As a major branch of machine learning, deep learning (DL) has been applied in physical layer communications as a potential solution to deal with the massive data and the high complexity of wireless communication systems [4]. By merging

Y. Yang and F. Gao are with Institute for Artificial Intelligence Tsinghua University (THUAI), State Key Lab of Intelligent Technologies and Systems, Beijing National Research Center for Information Science and Technology (BNRist), Department of Automation, Tsinghua University, Beijing, 100084, P. R. China (email: yyw18@mails.tsinghua.edu.cn, feifeigao@ieee.org).

C. Xing and J. An are with the School of Information and Electronics, Beijing Institute of Technology, Beijing 100081, China (e-mail: chengwenxing@ieee.org; an@bit.edu.cn).

A. Alkhateeb is with the School of Electrical, Computer and Energy Engineering at Arizona State University, Tempe, AZ 85287, USA (e-mail: alkhateeb@asu.edu).
DL into existing communication systems, many remarkable progresses have been made in various applications such as channel estimation [5]-[17], data detection [18], [19], channel feedback [20], [21], beamforming [22]-[24], and hybrid precoding [25], etc. In particular, conventional massive multipleinput multiple-output (MIMO) channel estimation algorithms have hit bottlenecks due to the prohibitively high overheads associated with massive antennas [26]-[29]. While DL based massive MIMO channel prediction can significantly improve the prediction accuracy or reduce the system overheads [11][17].

Compared with conventional communications that are based on statistics and information theories, DL based communications benefit from both the excellent learning capability of deep neural networks (DNNs) and the impressive computational throughput of parallel processing architectures. Besides, the most advantageous aspect of DL is its ability to handle problems with imperfect models or without mathematical models. Interestingly, there exists out-of-band side-information in communication systems that can be utilized to improve the system performance, including sub-6G channels, user positions, and 3D scene images obtained by cameras, etc. It should be noted that conventional communication techniques can hardly take advantage of these out-of-band side-information due to the lack of tractable mathematical models. In fact, the development of techniques that utilize out-of-band sideinformation to improve the system performance has been an emerging research trend in DL based communications. For example, [22] proposed a sub-6GHz channel information aided network for mmwave beam and blockage prediction, which could effectively reduce the overheads of both feedback and beam training. In [23], a novel 3D scene based beam selection architecture was developed for mmwave communications by using the surrounding 3D scene of the cellular coverage as the network inputs.

Meanwhile, model aided DL has also made much progress recently. Instead of purely relying on training data, model aided DL benefits from the guidance of model information and therefore can achieve better performance [3]. For instance, [10] proposed an efficient DL based channel estimator by learning the linear model between the least squares (LS) estimation and the linear minimum mean square error (LMMSE) estimation. In [19], the authors proposed a model-driven DL based MIMO detector by unfolding an iterative algorithm, which can significantly outperform the corresponding traditional iterative detector.

Although a few DL based works have tried to utilize multisource sensing information (MSI), e.g., out-of-band side- 
information and model information, to improve the system performance, none of them has yet investigated how to integrate and comprehensively utilize MSI in communication systems. Communication systems naturally work with multimodal data and this clear advantage should not be squandered. From the viewpoint of machine learning, data from various sources are referred as multimodal sensory data, whereas data from one source are referred as data of a single modality. Multimodal learning aims to build models that can fully exploit the constructive and complementary information lying in multimodal data, thus gaining performance advantages over methods that only use data of a single modality [30]. By combining DL architectures with multimodal learning methodologies, the concept of deep multimodal learning (DML) has been proposed in [31]. Thanks to the excellent flexibility of DL in extracting hierarchical features of data, DML offers several advantages over conventional multimodal learning, such as learning based feature extraction, implicit dimensionality reduction, and easily scalability in the modality number, etc [30]. So far, the mainstream applications of DML include human action recognition, audio-visual speaker detection, and autonomous driving, etc [30]-[32]. For example, [32] jointly exploited two modalities, i.e., image and optical flow, for human action recognition, which could obtain higher recognition accuracy than only using image data.

This paper aims to develop a systematic framework on DML based wireless communications. By using DML, the multimodal sensory data available in wireless communication systems can be fully exploited to provide constructive and complementary information for various tasks. The main contributions of this work can be summarized as following:

- We provide complete descriptions and heuristic analyses on the framework of DML based wireless communications. As opposed to [30] and [31] that mainly study DML in computer vision, speech, and natural language processing areas, this is the first work that explores how DML technologies can be applied to wireless communications to the best of the authors' knowledge.

- By investigating various modality combinations and fusion levels, we design several DML based architectures for channel prediction in massive MIMO systems as a case study. The design process presents a beneficial guidance on developing DML based communication technologies, while the proposed architectures can be easily extended to other communication problems like beam prediction, data detection, and antenna selection.

- Simulations based on ray-tracing software have been conducted and demonstrate that the proposed framework can effectively exploit the constructive and complementary information of multimodal sensory data in various scenarios.

The remainder of this paper is organized as follows. The framework of DML based wireless communications is given in Section III As a case study, Section III proposes several DML based architectures for channel prediction in massive MIMO systems. Numerical results are provided in Section IV followed by our main conclusions in Section $\mathrm{V}$
Notation: The bold letters denote vectors or matrices. The notation len $(\boldsymbol{z})$ denotes the length of the vector $\boldsymbol{x}$. The notations $(\cdot)^{T}$ and $(\cdot)^{H}$ respectively denote the transpose and the conjugate transpose of a matrix or a vector. The notation $\mathbb{C}^{m \times n}$ represents the $m \times n$ complex vector space. The notation $\|\boldsymbol{x}\|_{2}$ denotes the $L_{2}$ norm of $\boldsymbol{x}$. The notation $\circ$ represents the composite mapping operation. The notations $\Re[\cdot]$ and $\Im[\cdot]$, respectively, denote the real and imaginary parts of matrices, vectors or scales. The notations $*$ and $\otimes$ respectively represent the convolution operation and the matrix elementwise product. The notation $E[\cdot]$ represents the expectation with respect to all random variables within the brackets.

\section{DML FOR WIRELESS COMMUNICATIONS}

MSI in communication systems, including out-of-band sideinformation, model information, and other system information, is referred as multimodality. Information from one source is referred as one modality.

The framework of DML consists of three core parts: selection, extraction and fusion. The selection process is to select appropriate models as well as effective modalities for a certain task. The extraction process is to extract information from involved modalities. The fusion process is to fuse the extracted information in order to obtain a fused representation of the multimodal sensory data. To embrace the performance advantages offered by DML, there are several design choices to be considered, including the selections of models, modalities, fusion levels, and fusion strategies, as will be illustrated in the following.

\section{A. Model Selection}

DL models can generally be divided into two categories: discriminative and generative models. Discriminative models aim to learn the mapping function from the inputs to the outputs, and are typically used to solve regression and classification tasks. In other words, given the input $\boldsymbol{x}$ and the label $\boldsymbol{y}$, discriminative models learn the conditional probability $P(\boldsymbol{x} \mid \boldsymbol{y})$ by updating the model parameters. Since the majority of tasks in physical layer communications are to estimate $\boldsymbol{y}$ based on $\boldsymbol{x}$, such as channel estimation [6]-[8], data detection [18], [19], and beamforming [22]-[24], etc, existing DL techniques for physical layer communications mainly adopt discriminative models.

Generative models aim to learn the training data distribution that is required to generate new data with similar distributions. More specifically, generative models learn the joint probability $P(\boldsymbol{x}, \boldsymbol{y})$ in supervised learning problems, or learn the input data probability $P(\boldsymbol{x})$ in unsupervised or self-unsupervised learning problems. For DML problems, generative models are useful in the following three aspects: (1) Extracting features from different modalities; (2) Dealing with the situation of missing modalities during the test stage or lacking labeled data [33]; (3) Providing good initialization points for discriminative models, such as DNNs [34]. 


\section{B. Modality Selection}

Multimodal sensory data of communication systems typically have varying confidence level 1 when accomplishing different tasks. Take the beam selection at the base station (BS) as an example. The optimal beam vector can be obtained in three ways: (1) Finding the best beam based on known downlink channels [36]; (2) Learning from 3D scene images [23], [24]; (3) Extrapolating from sub-6G channels [22]. Among the three modalities, the downlink channels obviously have higher confidence level than both the 3D scene images and the sub-6G channels while the confidence level of the 3D scene images and the sub-6G channels depends on specifical scenarios. Nevertheless, even when we have access to the modality with the highest confidence level, there may exist some modalities that could provide complementary information to further improve the performance or robustness of the single-modality based methods [37]. To better understand this, we can refer to the maximum ratio combining (MRC) [38], which is a widely adopted technology to obtain the combining gains of multi-antenna communication systems. MRC could benefit from the antennas with worse channels, which also provides a revelatory understanding about the gains brought by modalities with relatively lower confidence levels.

Although different modalities could provide complementary information for a certain multimodal learning task, too much modalities may lead to information redundancy and an excessively complex fusion process. Therefore, it is worthwhile to select the optimal modalities by comprehensively considering the performance gain and the fusion complexity. In the area of computer vision and speech, the modality selection problem is generally considered as a tradeoff optimization [39]-[41]. For example, [39] proposed to select the optimal modalities based on the tradeoff between the feature dimensionality and the modality correlations. However, the authors did not take the confidence levels of modalities into account, which may miss modalities with high confidence levels. In [40], the authors utilized a dynamic programming approach to find the optimal subset of modalities based on the three-fold tradeoff between the performance gain, the overall confidence level of the selected subset, and the cost of the selected subset. In summary, [39]-[41] provide heuristic solutions for the modality selection problem in the context of multimedia data while there is hardly any literature studying modality selection for communication systems. A more direct way adopted in most of existing DML works is to manually select modalities by intuition and experiments.

\section{Fusion Level Selection}

Multimodal sensory data usually have different dimensionality and structures. For example, in massive MIMO systems, the user position data could be a $3 \times 1$ real-valued vector, the received signals could be a much longer complex-valued vector, and the transmitted signals could be a high-dimensional complex-valued matrix. To achieve efficient fusion, it is important to select proper modality fusion levels. In general, we

\footnotetext{
${ }^{1}$ The confidence level of one modality refers to the degree of the contribution or the reliability offered by the modality towards a certain task [35].
}

can perform the modality fusion in four levels: data fusion, feature fusion, decision fusion, or hybrid fusion.

1) Data fusion: Data fusion is to concatenate the raw or preprocessed data of all the modalities into a single vector and then to learn a joint multimodal representation based on the concatenated vector during the fusion, as shown in Fig. 1 (a). Data fusion is simple to design and allows end-toend training. An typical example of data fusion can refer to [8], where the received signals, the transmitted pilots, the pervious channels, and the LS estimates are directly concatenated as the inputs of networks to estimate channels in doubly selective fading scenarios. However, data fusion ignores the unique structure of different modalities, which may make it difficult to learn the complementary information among the modalities. In addition, simple concatenation of the multimodal sensory data leads to very high dimensional inputs, further resulting in the dimensionality curse problem 2 .

2) Feature fusion: Before we introduce the feature fusion, we first explain how to extract features from the raw or preprocessed data of one modality. The transform from the raw or preprocessed data to features is referred to as "feature extraction". Feature extraction algorithms are either generative or discriminative, linear or nonlinear, such as principal component analysis, linear discriminative analysis, and Laplacian eigenmaps, etc [43]. In recent few years, DNNs have been recognized as a popular technique to fuse modalities due to its excellent power and flexibility in extracting hierarchical features of the data. Specifically, each hidden layer of the network indeed represents a hierarchical features of the inputs. By changing the number of layers or choosing proper architecture, DNNs could extract features at various levels or with various dimensions. For example, [20] proposed a deep autoencoder architecture for channel feedback, where the dimension of the learnt compressed vector, i.e., the extracted features that are used to reconstruct the original channel, can be adjusted according to the given compression ratio.

Now, we discuss the feature fusion. As illustrated in Fig. 1 (b), feature fusion is to fuse higher-level features into a single hidden layer and then to learn a joint multimodal representations for the output. By utilizing the extracted higher-level features, the model with feature fusion could learn higherorder correlations across modalities. Moreover, thanks to the flexibility of feature dimension reduction offered by DNNs, the feature fusion strategy may have more advantages than the data fusion strategy in learning multimodal representations [30].

3) Decision fusion: Before we introduce decision fusion, we first explain how to acquire a decision for one modality. The process of obtaining task results based on the modal data is referred to as "decision acquirement". The decision acquirement can be realized by either DL based algorithms or conventional communication algorithms.

\footnotetext{
${ }^{2}$ The term "dimensionality curse" was first proposed in [42], which refers the phenomenon that when the data dimensionality increases, the dimension of feature space increases so fast that the available data become sparse and dissimilar in many ways. In this case, the amount of data required to support the data analysis often grows exponentially with the dimensionality.
} 


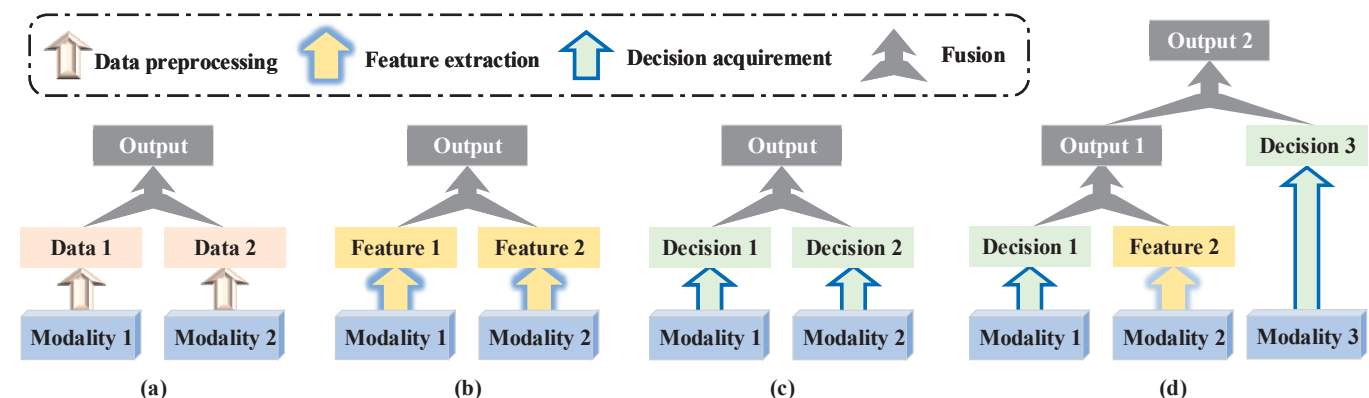

(a)

(b)

(c)

(d)

Fig. 1. Illustrations of various fusion levels for DML. (a) data fusion. (b) feature fusion. (c) decision fusion. (d) hybrid fusion.

TABLE I

ADVANTAGES AND DISADVANTAGES OF VARIOUS FUSION LEVELS

\begin{tabular}{|c|c|c|c|c|}
\hline Fusion levels & Data fusion & Feature fusion & Decision fusion & Hybrid fusion \\
\hline Disadvantage & $\begin{array}{l}\text { - ignore different modal- } \\
\text { ity structures } \\
\text {. dimensionality curse }\end{array}$ & $\begin{array}{l}\text { additional feature extraction } \\
\text { process }\end{array}$ & $\begin{array}{l}\text { cannot exploit feature } \\
\text { level correlations }\end{array}$ & - high design complexity \\
\hline Advantage & . easy to implement & - flexible dimension reduction & $\begin{array}{l}\text { - independent decisions } \\
\text {. easy to fuse }\end{array}$ & $\begin{array}{l}\text { - independent decisions } \\
\text { - flexible dimension reduction }\end{array}$ \\
\hline
\end{tabular}

As shown in Fig. 11(c), the decisions that are independently acquired by the involved modalities are fused to make a final decision, i.e., the output of the model. The disadvantage of decision fusion is that it cannot exploit the feature level correlations among modalities. The decision fusion strategy also has several advantages over the feature fusion strategy:

- When the involved modalities are completely uncorrelated or have very different dimensionality, it is much simpler and more reasonable to adopt decision fusion.

- Decision fusion makes it possible to adopt the most suitable algorithms to make decisions for each modality. In particular, for the modalities that can use accurate mathematical models to acquire the decisions, conventional communication theories based algorithms would be more suitable than DL based algorithms.

- The fusion task would be more easier to implement since the decisions of different modalities usually have similar data representations.

4) Hybrid fusion: To embrace the merits of both the feature and the decision fusion strategies, hybrid fusion combines both feature and decision fusion in a unified framework. Fig. 1(d) displays an example of hybrid fusion where the decisions and features of three modalities are fused at two different depths of the model. It should be emphasized that the decisions or features of multiple modalities can either be fused into a single layer or be fused gradually, i.e., modalities can be fused at different depths of the model. The choice at what depth to fuse which modalities is based on intuition and experiments. Take the channel estimation as an example. Given the three modalities, i.e., the pilots, the received signals, and the user position, we usually choose to first fuse the pilots and the received signals and then fuse the user position because the pilots and the received signals are highly correlated and the corresponding fusion should work well based on conventional communication theories. Besides, the gradual fusion strategy could also avoid overlarge fusion vectors, which partially solves the problem of dimensionality curse.

Tab. I lists the advantages and disadvantages of various fusion levels. So far, only the data fusion has been utilized in wireless communications, referring to [8]. It should be mentioned that the fusion level selection depends on the specifical problem, and therefore the superiority of the fusion level strategies should be investigated in a specific problem rather than in an absolute sense.

\section{Fusion Strategy Selection}

Various methods can be used to fuse different modalities, among which fixed-rule based fusion is the simplest one, including "max", "min", "average", and "majority voting", etc (see more rules in [44|). Besides, the linear weighted is also a common fusion strategy, where features or decisions of different modalities are combined with linear weights. One successful application of linear weighted is MRC, where the weights can be directly determined by channels. However, the linear weighted based modality fusion is not so simple like MRC. The greatest challenge lies in the determination of the weights for each modality, especially when the data dimension is high. To solve this problem, DNN based fusion has been proposed and gained growing attentions in these years [31], [32]. DNN based fusion could learn a nonlinear weighted mapping from the input to the output, and the weights could be adjusted by training with pre-acquired datasets instead of manual selection.

\section{Case Study: DML for Massive MiMO Channel PREDICTION}

In this section, we will first present the available modalities for channel prediction in massive MIMO systems. We will also give brief descriptions of the involved network architectures. Then, we will respectively discuss the architecture designs for the BS and the user, followed by detailed training steps of the proposed networks. 


\section{A. Available Modalities for Channel Prediction}

The acquisition of the channel knowledge plays a critical role in massive MIMO which is a promising technology for future wireless communication systems [26], [45]. In this work, we consider a massive MIMO system, where a BS is equipped with $M \gg 1$ antennas in the form of uniform linear array (ULA) 3 and serves multiple single-antenna users. Note that the proposed approaches are applicable for uplink/downlink channel prediction in both time division duplex (TDD) and frequency division duplex (FDD) systems. Due to the prohibitively high overheads associated with downlink training and uplink feedback, the downlink channel prediction in FDD massive MIMO system is an extremely challenging task [12][14]. Therefore, we take the downlink channel prediction in FDD massive MIMO system as an typical example to illustrate the design and application of DML based channel prediction.

System model: To discuss the available modalities in FDD massive MIMO systems, we first present the mathematical model for the downlink transmission. Denote $T_{p}$ as the pilot length. The received frequency domain signal of the $u$-th user on the $k$-th subcarrier is

$$
\boldsymbol{r}[k]=\boldsymbol{h}_{u}^{T}\left(f_{\mathrm{D}, k}\right) \boldsymbol{s}[k]+\boldsymbol{\varepsilon}[k],
$$

where $\boldsymbol{r}[k] \in \mathbb{C}^{1 \times T_{p}}$ is the received signal, $\boldsymbol{s}[k] \in \mathbb{C}^{M \times T_{p}}$ is the downlink pilot signal, $\varepsilon[k]$ is the additive white Gaussian noise. Moreover, $\boldsymbol{h}_{u}\left(f_{\mathrm{D}, k}\right) \in \mathbb{C}^{M \times 1}$ is the downlink channel that can be written as [46]

$$
\boldsymbol{h}_{u}\left(f_{\mathrm{D}, k}\right)=\sum_{p=1}^{P} \alpha_{u, p} e^{-j 2 \pi f_{\mathrm{D}, k} \tau_{u, p}+j \phi_{u, p}} \boldsymbol{a}\left(\theta_{\mathrm{az}}^{u, p}, \theta_{\mathrm{el}}^{u, p}\right),
$$

where $P$ is the path number, $f_{\mathrm{D}, k}$ is the frequency of the $k$-th downlink subcarrier, while $\alpha_{u, p}, \phi_{u, p}$, and $\tau_{u, p}$ are the attenuation, phase shift, and delay of the $p$-th path, respectively. In addition, $\boldsymbol{a}\left(\theta_{\mathrm{az}}^{u, p}, \theta_{\mathrm{el}}^{u, p}\right)$ is the array manifold vector defined as

$$
\begin{aligned}
\boldsymbol{a}\left(\theta_{\mathrm{az}}^{u, p}, \theta_{\mathrm{el}}^{u, p}\right)=[ & 1, e^{j \varpi \sin \left(\theta_{\mathrm{el}}^{u, p}\right) \cos \left(\theta_{\mathrm{az}}^{u, p}\right)}, \ldots \\
& \left.\ldots, e^{j \varpi(M-1) \sin \left(\theta_{\mathrm{el}}^{u, p}\right) \cos \left(\theta_{\mathrm{az}}^{u, p}\right)}\right]^{T},
\end{aligned}
$$

where $\varpi=2 \pi d f_{\mathrm{D}, k} / c, d$ is the antenna spacing, $c$ is the speed of light, and $\left\{\theta_{\mathrm{az}}^{u, p}, \theta_{\mathrm{el}}^{u, p}\right\}$ is the \{azimuth, elevation angle of arrival. We employ the accurate $3 \mathrm{D}$ ray-tracing simulator Wireless InSite [46] to obtain the channel parameters in Eq. (2), i.e., $\left\{\alpha_{u, p}, \phi_{u, p}, \tau_{u, p}, \theta_{\mathrm{az}}^{u, p}, \theta_{\mathrm{el}}^{u, p}\right\}$. To simplify the notation, we drop the sub-carrier index $k$ and the user index $u$ in the rest of the paper, e.g., replacing $\boldsymbol{r}[k], \boldsymbol{h}_{u}\left(f_{\mathrm{D}, k}\right)$, and $\boldsymbol{s}[k]$ with $\boldsymbol{r}, \boldsymbol{h}\left(f_{\mathrm{D}}\right)$, and $\boldsymbol{s}$, respectively.

Available Modality: Available modalities in FDD massive MIMO system could be the received signals, the pilots, the LS estimate, the downlink channels of previous coherent time periods, the uplink channel, the user location, and the partial downlink channel, as described in the following.

1) Received signals and pilots: Eq. (1) indeed reveals that there exists a mapping function from $\{\boldsymbol{r}, \boldsymbol{s}\}$ to $\boldsymbol{h}\left(f_{\mathrm{D}}\right)$, which

\footnotetext{
${ }^{3}$ We adopt the ULA model here for simpler illustration, nevertheless, the proposed approaches are not restricted to the specifical array shape, and therefore are applicable for array with arbitrary geometry.
}

indicates that the received signals $r$ and the pilots $s$ are two modalities that could be jointly utilized to predict the downlink channel $\boldsymbol{h}\left(f_{\mathrm{D}}\right)$.

2) LS estimate: When the number of pilots are sufficient (i.e., $\left.T_{p} \geq M\right), \boldsymbol{h}\left(f_{\mathrm{D}}\right)$ can be estimated by LS [47], i.e.,

$$
\hat{\boldsymbol{h}}_{\mathrm{LS}}\left(f_{\mathrm{D}}\right)=\boldsymbol{r} \boldsymbol{s}^{H}\left(s \boldsymbol{s}^{H}\right)^{-1} \text {. }
$$

In fact, the LS estimate $\hat{\boldsymbol{h}}_{\mathrm{LS}}\left(f_{\mathrm{D}}\right)$ can be regarded as one modality from model information.

3) Previous downlink channels: Denote the superscript $(\tau)$ as the index of coherent time periods. The downlink channels of previous coherent time periods, i.e., $\boldsymbol{h}^{(\tau-1)}\left(f_{\mathrm{D}}\right), \boldsymbol{h}^{(\tau-2)}\left(f_{\mathrm{D}}\right), \cdots$, are referred as previous downlink channels, $\overleftarrow{\boldsymbol{h}}\left(f_{\mathrm{D}}\right)$, for ease of exposition 4 . In practical systems, there exist unknown time correlations among channels that cannot be exploited by conventional channel estimation algorithms. Whereas such time correlations could be implicitly learned by DNNs and then be used to improve the prediction accuracy [8], [9], [11].

4) User location: The user location can be obtained by various techniques, such as the ultra-wideband, the global positioning system, and the wireless fidelity, etc. Many positioning works have revealed that there is a distinct link between the user's position and channels [48]. Define the locationto-channel mapping as $\boldsymbol{\Phi}_{f}:\left\{\boldsymbol{D}_{(x, y, z)}\right\} \rightarrow\{\boldsymbol{h}(f)\}$, where $\boldsymbol{D}_{(x, y, z)}$ is the 3D coordinate of the user, and $f$ is the carrier frequency. Based on the universal approximation theorem [49] and the widely adopted assumption that $\boldsymbol{\Phi}_{f}$ is a bijective deterministic mapping in massive MIMO systems [48], we know that the mapping function $\boldsymbol{\Phi}_{f}$ could be approximated arbitrarily well by a DNN under ideal conditions. Therefore, the modality of user location could be adopted to predict the downlink channel by using DNNs to learn the mapping $\boldsymbol{\Phi}_{f_{\mathrm{D}}}$.

5) Uplink channel: Since uplink channels are easier to obtain than downlink channels in massive MIMO systems, many studies utilize uplink channels to aid the downlink channel prediction [13], [14], [50]. With the assumption that $\boldsymbol{\Phi}_{f}$ is a bijective deterministic mapping, the channel-to-location mapping $\boldsymbol{\Phi}_{f}^{-1}$ exists and can be written as $\boldsymbol{\Phi}_{f}^{-1}:\{\boldsymbol{h}(f)\} \rightarrow$ $\left\{\boldsymbol{D}_{(x, y, z)}\right\}$. Hence, the uplink-to-downlink mapping $\boldsymbol{\Psi}_{\mathrm{U} \rightarrow \mathrm{D}}$ exists, and can be written as follows [13]:

$$
\boldsymbol{\Psi}_{\mathrm{U} \rightarrow \mathrm{D}}=\boldsymbol{\Phi}_{f_{\mathrm{D}}} \circ \boldsymbol{\Phi}_{f_{\mathrm{U}}}^{-1}:\left\{\boldsymbol{h}\left(f_{\mathrm{U}}\right)\right\} \rightarrow\left\{\boldsymbol{h}\left(f_{\mathrm{D}}\right)\right\},
$$

where $f_{\mathrm{U}}$ is the uplink frequency, and $\boldsymbol{\Phi}_{f_{\mathrm{D}}} \circ \boldsymbol{\Phi}_{f_{\mathrm{U}}}^{-1}$ represents the composite mapping related to $\boldsymbol{\Phi}_{f_{\mathrm{D}}}$ and $\boldsymbol{\Phi}_{f_{\mathrm{U}}}^{-1}$. Therefore, the modality of uplink channel could also be adopted to predict the downlink channel by using DNNs to learn the mapping $\Psi_{\mathrm{U} \rightarrow \mathrm{D}}$.

6) Partial downlink channel: Due to the high cost and power consumption of the radio-frequency chains, massive MIMO systems usually adopt hybrid analog and digital transceivers that are operated with switchers [25]. Therefore, given the limited transmission period and pilot length, only partial downlink channel can be obtained by the user and then

\footnotetext{
${ }^{4}$ Since the downlink channel to be predicted and other modalities involved are all the data in the $\tau$-th coherent time period, we have omitted the superscript $(\tau)$ of these realtime data for simplicity.
} 
TABLE II

MODALITIES INVOLVED IN DOWNLINK CHANNEL PREDICTION

\begin{tabular}{|c|c|c|c|c|c|c|}
\hline Modality & $\{r, s\}$ & $\hat{h}_{\mathrm{LS}}\left(f_{\mathrm{D}}\right)[\mathbf{8}],[10]$ & $\overleftarrow{h}\left(f_{\mathrm{D}}\right)[\mathbf{V}],[9]$ & $D_{(x, y, z)}$ & $\boldsymbol{h}\left(f_{\mathrm{U}}\right)[\mathbf{1 2}]-[14]$ & $\breve{h}\left(f_{\mathrm{D}}\right)[\mathbf{1 3}],[17]$ \\
\hline BS side & $\sqrt{ }$ & $\times$ & $\sqrt{ }$ & $\sqrt{ }$ & $\sqrt{ }$ & $\sqrt{ }$ \\
User side & $\sqrt{ }$ & $\sqrt{ }$ & $\sqrt{ }$ & $\sqrt{ }$ & $\times$ & $\sqrt{ }$ \\
\hline
\end{tabular}

be fed back to BS. Denote the known partial downlink channel as $\breve{\boldsymbol{h}}\left(f_{\mathrm{D}}\right)$ with len $\left(\breve{\boldsymbol{h}}\left(f_{\mathrm{D}}\right)\right)<M$. Denote the vector consisting of unknown elements in $\boldsymbol{h}\left(f_{\mathrm{D}}\right)$ as $\widehat{\boldsymbol{h}}\left(f_{\mathrm{D}}\right)$. Recalling Eq. (3) and Eq. (2), it is obvious that there exists a deterministic mapping from $\breve{\boldsymbol{h}}\left(f_{\mathrm{D}}\right)$ to $\widehat{\boldsymbol{h}}\left(f_{\mathrm{D}}\right)$, which can be written as $\boldsymbol{\Upsilon}:\left\{\widetilde{\boldsymbol{h}}\left(f_{\mathrm{D}}\right)\right\} \rightarrow\left\{\widehat{\boldsymbol{h}}\left(f_{\mathrm{D}}\right)\right\}$. Therefore, we can predict the downlink channel by learning the mapping $\Upsilon$.

In order to facilitate the analysis, we list the modalities for downlink channel prediction in Tab. II where " $\sqrt{ }$ " and " $\times$ " respectively represent the available and unavailable modalities for BS or the user. In particular, the modalities $\{\boldsymbol{r}, s\}$ and $\breve{\boldsymbol{h}}\left(f_{\mathrm{D}}\right)$ are available for BS because $\boldsymbol{r}$ and $\breve{\boldsymbol{h}}\left(f_{\mathrm{D}}\right)$ could be fed back to the BS by the user. The modality $\hat{\boldsymbol{h}}_{\mathrm{LS}}\left(f_{\mathrm{D}}\right)$ is obtained based on $\{\boldsymbol{r}, \boldsymbol{s}\}$. When the length of $\boldsymbol{r}$ is sufficiently long for the LS estimator, i.e., $T_{p} \geq M$, it would be more efficient to directly feed back the downlink channel rather than $\boldsymbol{r}$ to BS. Therefore, we set the modality $\hat{\boldsymbol{h}}_{\mathrm{LS}}\left(f_{\mathrm{D}}\right)$ to be unavailable at BS. Tab. $\Pi$ also displays the existing works that utilize aforementioned modalities to predict channels. By trying and testing possible modality combinations and feature level strategies, we can find the modalities with higher confidence levels and the modality combinations with better performance.

\section{B. DNN Architectures}

Based on the definition in Section III-A, the downlink channel prediction is a typical discriminative regression task. Since discriminative models are naturally suitable for feature extraction and decision acquirement in discriminative tasks, we choose discriminative models for downlink CSI prediction. The selections of both modalities and feature level strategies depends on specifical scenarios. Besides, due to the excellent learning capability of DNNs, we adopt DNN based fusion for channel prediction rather than fixed-rule based fusion.

Activation function: The activation functions, including leaky rectified linear units (LeakyReLU) 5 , Sigmoid, and Tanh, apply element-wise nonlinear transformations to the outputs of the network layers. The functions LeakyReLU, Sigmoid, and Tanh can be respectively written as $\mathcal{F}_{\mathrm{LR}}(\boldsymbol{x})=\max \{\boldsymbol{x}, 0.2 \boldsymbol{x}\}$, $\mathcal{F}_{\mathrm{Sig}}(\boldsymbol{x})=1 /\left(1+e^{-\boldsymbol{x}}\right)$, and $\mathcal{F}_{\mathrm{Tan}}(\boldsymbol{x})=\left(e^{\boldsymbol{x}}-e^{-\boldsymbol{x}}\right) /\left(e^{\boldsymbol{x}}+\right.$ $\left.e^{-\boldsymbol{x}}\right)$.

Loss function: A DNN architecture consists of the input $\boldsymbol{x}$, the label $\boldsymbol{y}$, the output $\hat{\boldsymbol{y}}$, the network parameter $\boldsymbol{\Omega}$, activation functions, the loss function $\operatorname{Loss}(\boldsymbol{\Omega})$, a back-propagation learning algorithm, and network layers. Specifically, the net-

\footnotetext{
${ }^{5}$ We adopt LeakyReLU instead of normal rectified linear units (ReLU) to avoid the "dead Relu" phenomenon [51].
}

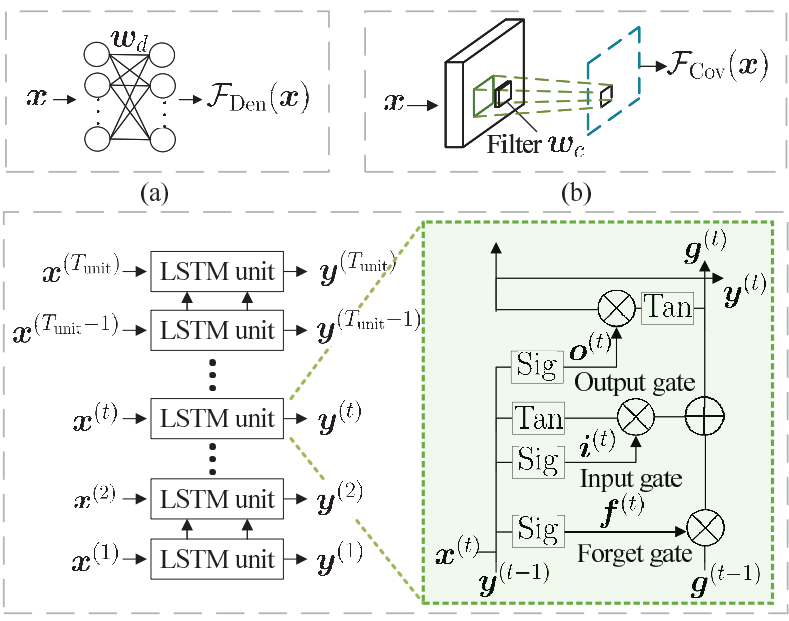

(c)

Fig. 2. Illustrations of the dense layer (a), the convolution layer (b), and the LSTM layer (c).

work parameter $\Omega$ includes the weights and the biases of the network layers. The loss function adopted in this work is

$$
\operatorname{Loss}(\boldsymbol{\Omega})=\frac{1}{V} \sum_{v=0}^{V-1}\left\|\hat{\boldsymbol{y}}_{v}-\boldsymbol{y}_{v}\right\|_{2}^{2},
$$

where $V$ is the batch size, and the subscript $v$ denotes the index of the $v$-th training sample. The back-propagation learning algorithm adopted in this work is the adaptive moment estimation (ADAM) algorithm [52]. In the off-line training stage, the network parameter $\Omega$ is updated by the ADAM algorithm to minimize the loss function Loss $(\boldsymbol{\Omega})$ on the training dataset. While in the on-line testing stage, $\Omega$ is fixed and the network could directly output the estimates of the labels in the testing dataset with a rather small error.

Network layer: Fig. 2 depicts the structure of the network layers, including the dense, the convolution and the LSTM layers. As shown in Fig. 2 (a), the dense layer can be mathematically expressed as $\mathcal{F}_{\text {Den }}(\boldsymbol{x})=\boldsymbol{w}_{d} \boldsymbol{x}+\boldsymbol{b}_{d}$, where $\boldsymbol{w}_{d}$ and $\boldsymbol{b}_{d}$ are the weight and the bias of the dense layer, respectively. Compared with the dense layer, the convolution layer is more powerful in learning the spatial features of the inputs. As illustrated in Fig. 2 (b), the convolution layer can be mathematically expressed as $\mathcal{F}_{\mathrm{Cov}}(\boldsymbol{x})=\boldsymbol{w}_{c} * \boldsymbol{x}+\boldsymbol{b}_{c}$, where $\boldsymbol{w}_{c}$ and $\boldsymbol{b}_{c}$ is the weight and the bias of the filter, respectively. Fig. 2 (c) depicts the structure of the LSTM layer, where each LSTM layer contains $T_{\text {unit }}$ LSTM units. The output of the LSTM layer can be written as

$$
\mathcal{F}_{\text {LSTM }}\left(\boldsymbol{x}^{(1)}, \cdots, \boldsymbol{x}^{\left(T_{\text {unit }}\right)}\right)=\left(\boldsymbol{y}^{(1)}, \cdots, \boldsymbol{y}^{\left(T_{\text {unit }}\right)}\right) .
$$

In the $t$-th $\left(1 \leq t \leq T_{\text {unit }}\right)$ LSTM units, the relationships 


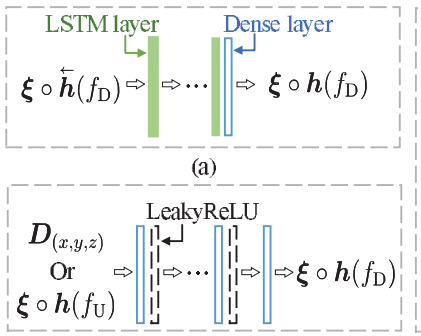

(b)

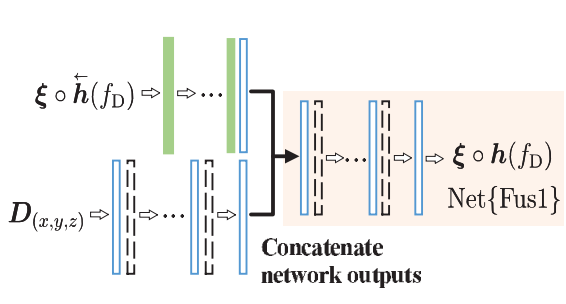

(c)

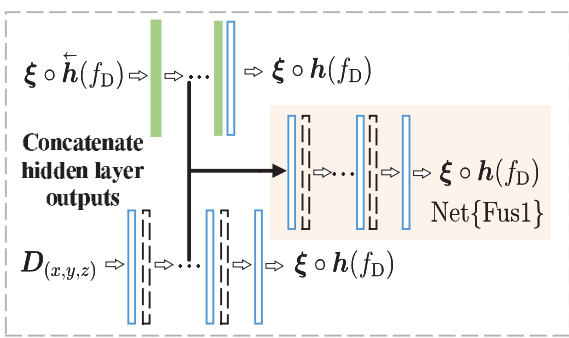

(d)

Fig. 3. The network structures of $\operatorname{Net}\{H\}$ (a), $\operatorname{Net}\{L\}$ (b), $\operatorname{Net}\{\mathrm{U}\}$ (b), $\operatorname{Net}\{\mathrm{H}, \mathrm{L}\}_{\mathrm{d}}$ (c), and $\operatorname{Net}\{\mathrm{H}, \mathrm{L}\}_{\mathrm{f}}$ (d).

between the input $\boldsymbol{x}^{(t)}$ and output $\boldsymbol{y}^{(t)}$ can be expressed with the following equations:

$$
\begin{aligned}
\boldsymbol{i}^{(t)}= & \mathcal{F}_{\mathrm{Sig}}\left(\boldsymbol{w}_{i}\left[\boldsymbol{y}^{(t-1)}, \boldsymbol{x}^{(t)}\right]+\boldsymbol{b}_{i}\right) ; \\
\boldsymbol{f}^{(t)}= & \mathcal{F}_{\mathrm{Sig}}\left(\boldsymbol{w}_{f}\left[\boldsymbol{y}^{(t-1)}, \boldsymbol{x}^{(t)}\right]+\boldsymbol{b}_{f}\right) ; \\
\boldsymbol{o}^{(t)}= & \mathcal{F}_{\mathrm{Sig}}\left(\boldsymbol{w}_{o}\left[\boldsymbol{y}^{(t-1)}, \boldsymbol{x}^{(t)}\right]+\boldsymbol{b}_{o}\right) ; \\
\boldsymbol{g}^{(t)}= & \boldsymbol{f}^{(t)} \otimes \boldsymbol{g}^{(t-1)}+ \\
& \boldsymbol{i}^{(t)} \otimes \mathcal{F}_{\operatorname{Tan}}\left(\boldsymbol{w}_{g}\left[\boldsymbol{y}^{(t-1)}, \boldsymbol{x}^{(t)}\right]+\boldsymbol{b}_{g}\right) ; \\
\boldsymbol{y}^{(t)=} & \boldsymbol{o}^{(t)} \otimes \mathcal{F}_{\operatorname{Tan}}\left(\boldsymbol{g}^{(t)}\right),
\end{aligned}
$$

where $\left\{\boldsymbol{w}_{i}, \boldsymbol{w}_{f}, \boldsymbol{w}_{o}, \boldsymbol{w}_{g}\right\}$ and $\left\{\boldsymbol{b}_{i}, \boldsymbol{b}_{f}, \boldsymbol{b}_{o}, \boldsymbol{b}_{g}\right\}$ are respectively the weights and the biases of the LSTM units, while $\boldsymbol{i}^{(t)}$, $\boldsymbol{f}^{(t)}$ and $\boldsymbol{o}^{(t)}$ are respectively the input gate, the forget gate and output gate. Moreover, $\boldsymbol{g}^{(t)}$ is the cell state of the $t$-th LSTM unit. Since the LSTM layer can effectively learn both the short-term and the long-term features through the memory cell and the gates, it has been recognized as a useful tool for time series related tasks.

\section{Architecture Designs at the BS Side}

Accurate downlink channels are crucial for BS to obtain high beamforming gains. Here we consider the downlink channel prediction problem under two different scenarios, i.e., feedback link being unavailable or available. Before coming to specifical architectures, we first present our main idea to design fusion architectures as follow:

(i) Design and train elementary networks, i.e., the networks that adopt as few as possible modalities to independently predict downlink channels. In fact, all the modalities listed in Tab. II can independently predict downlink channels except the two modalities $\{\boldsymbol{r}, \boldsymbol{s}\}$ that should be jointly utilized to obtain downlink channels. Note that the performance of the elementary networks can be used to measure the confidence levels of the corresponding modalities.

(ii) Design and train two-element based networks, i.e., the networks that fuse two elementary networks. The performance of the two-element based networks can be used to measure the complementarity of the corresponding modality combinations. When we design fusion architectures with multiple modalities, we will preferentially fuse the modality combinations with better performance and then fuse the modalities with higher confidence levels based on experiments and intuition [30].

The idea is also applicable to the architecture designs at the user side as will be shown in the later section.

1) Feedback link is unavailable: In this scenario, available modalities are the previous downlink channels $\overleftarrow{\boldsymbol{h}}\left(f_{\mathrm{D}}\right)$, the user location $\boldsymbol{D}_{(x, y, z)}$, and the uplink channel $\boldsymbol{h}\left(f_{\mathrm{U}}\right)$. To investigate the confidence levels of the three modalities, we propose three networks, i.e., $\operatorname{Net}\{\mathrm{H}\}, \operatorname{Net}\{\mathrm{L}\}$, and $\operatorname{Net}\{\mathrm{U}\}$ to respectively predict the downlink channel based on the previous downlink channels, the user location, and the uplink channel. Fig. 3 (a) illustrates the network structure of $\operatorname{Net}\{\mathrm{H}\}$. The input of $\operatorname{Net}\{\mathrm{H}\}$ is $\boldsymbol{\xi} \circ \overleftarrow{\boldsymbol{h}}\left(f_{\mathrm{D}}\right)$, where

$$
\overleftarrow{\boldsymbol{h}}\left(f_{\mathrm{D}}\right)=\left[\boldsymbol{h}^{(\tau-1)}\left(f_{\mathrm{D}}\right), \cdots, \boldsymbol{h}^{\left(\tau-T_{\mathrm{unit}}\right)}\left(f_{\mathrm{D}}\right)\right]
$$

while $\boldsymbol{\xi}$ is the mapping between the complex and the real domains, i.e., $\boldsymbol{\xi}: \boldsymbol{z} \rightarrow\left(\Re\left(\boldsymbol{z}^{T}\right), \Im\left(\boldsymbol{z}^{T}\right)\right)^{T}$. The label of $\operatorname{Net}\{\mathrm{H}\}$ is $\boldsymbol{\xi} \circ \boldsymbol{h}\left(f_{\mathrm{D}}\right)$. The network $\operatorname{Net}\{\mathrm{H}\}$ is composed of several LSTM layers and one dense layer. Here we adopt the LSTM layer to predict the downlink channels for its superiority in time series data analyses. Besides, we add the dense layer after the last LSTM layer is to release the output of $\operatorname{Net}\{\mathrm{H}\}$ from the limited value range of the activation functions $\mathcal{F}_{\text {Tan }}$ and $\mathcal{F}_{\text {Sig }}$, as indicated in Eq. (7e). Fig. 3 (b) shows the network structure of both $\operatorname{Net}\{\mathrm{L}\}$ and $\operatorname{Net}\{\mathrm{U}\}$, where the network is composed of several dense layers, and each dense layer except for the output layer is followed by the LeakyReLU function. Note that $\operatorname{Net}\{L\}$ and Net $\{U\}$ have the common network structure and the same label $\boldsymbol{\xi} \circ \boldsymbol{h}\left(f_{\mathrm{D}}\right)$, but they have different inputs and different hype-parameters, including the number of layers, the number of neurons in each layers, and the learning rates, etc.

To investigate the complementarities of the three modalities i.e., $\overleftarrow{\boldsymbol{h}}\left(f_{\mathrm{D}}\right), \boldsymbol{D}_{(x, y, z)}$, and $\boldsymbol{h}\left(f_{\mathrm{U}}\right)$, we first propose Net $\{\mathrm{L}, \mathrm{U}\}_{\mathrm{d}}$ $\operatorname{Net}\{\mathrm{H}, \mathrm{U}\}_{\mathrm{d}}$, and $\operatorname{Net}\{\mathrm{H}, \mathrm{L}\}_{\mathrm{d}}$ to respectively fuse two of the three modalities at the decision levels. As shown in Fig. 3 (c), $\operatorname{Net}\{\mathrm{H}, \mathrm{L}\}_{\mathrm{d}}$ consists of $\operatorname{Net}\{\mathrm{H}\}, \operatorname{Net}\{\mathrm{L}\}$, and $\operatorname{Net}\{\mathrm{Fus} 1\}$. The network Net\{Fus1\}, composed of several dense layers and LeakyReLU functions, concatenates the network outputs of $\operatorname{Net}\{\mathrm{H}\}$ and $\operatorname{Net}\{\mathrm{L}\}$ as its input vector. Note that the structures of $\operatorname{Net}\{\mathrm{L}, \mathrm{U}\}_{\mathrm{d}}$ and $\operatorname{Net}\{\mathrm{H}, \mathrm{U}\}_{\mathrm{d}}$ can be similarly obtained following the design of $\operatorname{Net}\{\mathrm{H}, \mathrm{L}\}_{\mathrm{d}}$. Therefore, we omit the descriptions of these networks for simplicity. Then, we propose $\operatorname{Net}\{\mathrm{L}, \mathrm{U}\}_{\mathrm{f}}, \operatorname{Net}\{\mathrm{H}, \mathrm{U}\}_{\mathrm{f}}$, and $\operatorname{Net}\{\mathrm{H}, \mathrm{L}\}_{\mathrm{f}}$ to respec- 


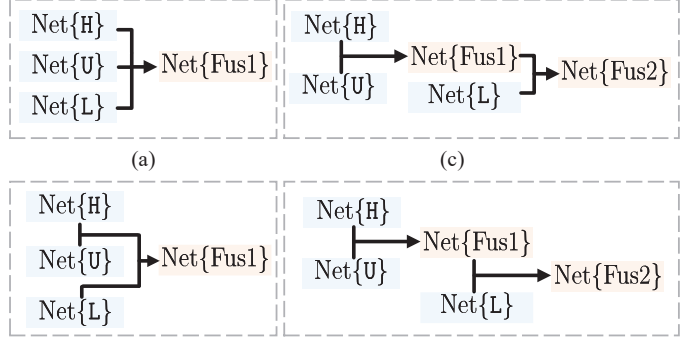

(b)

(d)

Fig. 4. The network structures of $\operatorname{Net}\{\mathrm{H}, \mathrm{L}, \mathrm{U}\}_{\mathrm{d}}$ (a), $\operatorname{Net}\{\mathrm{H}, \mathrm{L}, \mathrm{U}\}_{\mathrm{f}}$ (b), $\operatorname{Net}\{\mathrm{H}, \mathrm{L}, \mathrm{U}\}_{\mathrm{h} 1}(\mathrm{c})$, and $\operatorname{Net}\{\mathrm{H}, \mathrm{L}, \mathrm{U}\}_{\mathrm{h} 2}(\mathrm{~d})$.

tively fuse two of the three modalities at the feature levels. As shown in Fig. 3 (d), the main difference between $\operatorname{Net}\{\mathrm{H}, \mathrm{L}\}_{\mathrm{f}}$ and $\operatorname{Net}\{\mathrm{H}, \mathrm{L}\}_{\mathrm{d}}$ is that $\operatorname{Net}\{\mathrm{H}, \mathrm{L}\}_{\mathrm{f}}$ concatenates the hidden layer outputs rather than the network outputs of $\operatorname{Net}\{\mathrm{H}\}$ and $\operatorname{Net}\{L\}$. Similarly, we omit the descriptions of $\operatorname{Net}\{L, U\}_{f}$ and Net $\{\mathrm{H}, \mathrm{U}\}$ f for simplicity. It should be explained that we do not consider data level fusion for $\overleftarrow{\boldsymbol{h}}\left(f_{\mathrm{D}}\right), \boldsymbol{D}_{(x, y, z)}$, and $\boldsymbol{h}\left(f_{\mathrm{U}}\right)$ since the three modalities have very different dimensions and data structures, which would result in inefficient data fusion.

Furthermore, we propose $\operatorname{Net}\{\mathrm{H}, \mathrm{L}, \mathrm{U}\}_{\mathrm{d}}$ and $\operatorname{Net}\{\mathrm{H}, \mathrm{L}, \mathrm{U}\}_{\mathrm{f}}$ to fuse all the three modalities at the decision and the feature levels, respectively. As illustrated in Fig. 4 (a) and Fig. 4 (b), Net $\{\mathrm{H}, \mathrm{L}, \mathrm{U}\}_{\mathrm{d}}$ and $\operatorname{Net}\{\mathrm{H}, \mathrm{L}, \mathrm{U}\}_{\mathrm{f}}$ are both composed of $\operatorname{Net}\{\mathrm{H}\}, \operatorname{Net}\{\mathrm{L}\}, \operatorname{Net}\{\mathrm{U}\}$, and Net $\{$ Fus1 $\}$. The difference between $\operatorname{Net}\{\mathrm{H}, \mathrm{L}, \mathrm{U}\}_{\mathrm{d}}$ and $\operatorname{Net}\{\mathrm{H}, \mathrm{L}, \mathrm{U}\}_{\mathrm{f}}$ is that $\operatorname{Net}\{\mathrm{H}, \mathrm{L}, \mathrm{U}\}_{\mathrm{f}}$ concatenates all the hidden layer outputs rather than the network outputs of $\operatorname{Net}\{H\}, \operatorname{Net}\{L\}$, and $\operatorname{Net}\{\mathrm{U}\}$. Moreover, we propose $\operatorname{Net}\{\mathrm{H}, \mathrm{L}, \mathrm{U}\}_{\mathrm{h} 1}$ and $\operatorname{Net}\{\mathrm{H}, \mathrm{L}, \mathrm{U}\}_{\mathrm{h} 2}$ to fuse all the three modalities at the hybrid level. As depicted in Fig. 4 (c), $\operatorname{Net}\{\mathrm{H}, \mathrm{L}, \mathrm{U}\}_{\mathrm{h} 1}$ first uses Net $\{$ Fus 1$\}$ to fuse the hidden layer outputs of $\operatorname{Net}\{\mathrm{H}\}$ and $\operatorname{Net}\{\mathrm{U}\}$ and then uses Net Fus2 $\}$ to fuse the network output of Net $\{$ Fus 1$\}$ and the hidden layer output of Net $\{L\}$. The only difference between $\operatorname{Net}\{H, L, U\} h 1$ and $\operatorname{Net}\{\mathrm{H}, \mathrm{L}, \mathrm{U}\}_{\mathrm{h} 2}$ is that $\operatorname{Net}\{\mathrm{H}, \mathrm{L}, \mathrm{U}\}_{\mathrm{h} 1}$ fuses the network outputs of both Net Fus1\} and $\operatorname{Net}\{\mathrm{L}\}$ while $\operatorname{Net}\{\mathrm{H}, \mathrm{L}, \mathrm{U}\}_{\mathrm{h} 2}$ fuses the hidden layer outputs of both Net Fus1 $\}$ and $\operatorname{Net}\{\mathrm{L}\}$. It should be mentioned that we choose to first fuse $\operatorname{Net}\{\mathrm{H}\}$ and $\operatorname{Net}\{\mathrm{U}\}$ at the feature level because $\operatorname{Net}\{\mathrm{H}, \mathrm{U}\}_{\mathrm{f}}$ outperforms other proposed two-modality based networks, as will be shown in the later simulation section. This indicates that the fusion of $\overleftarrow{\boldsymbol{h}}\left(f_{\mathrm{D}}\right)$ and $\boldsymbol{h}\left(f_{\mathrm{U}}\right)$ provides stronger complementarity and therefore would be more suitable to be fused earlier. Note that the design and the testing for DML are not isolated but interoperable, which means that we need the testing results to guide the design of network. In other words, the excellent capability and flexibility of DML come at the cost of design complexity.

Remark 1: The channel prediction based on the three modalities, i.e., $\overleftarrow{\boldsymbol{h}}\left(f_{\mathrm{D}}\right), \boldsymbol{D}_{(x, y, z)}$, and $\boldsymbol{h}\left(f_{\mathrm{U}}\right)$, can also be referred as the channel extrapolation information across the time, space, and frequency domains. The three-modality based networks in Fig. 4 jointly exploit the complementarity of the time-space-frequency information to improve the performance of the channel extrapolation.
2) Feedback link is available: In this scenario, we need to investigate which modality would be more efficient to be fed back to BS under given feedback overhead. When the length of the vector to be fed back, denoted by $T_{\mathrm{fb}}$, is greater than the number of BS antennas $M$, it is obvious that we should directly feed back the downlink channel rather than the received signal $\boldsymbol{r}$. When $T_{\mathrm{fb}}$ is smaller than $M$, we respectively try various fusion networks for $\{\boldsymbol{r}, \boldsymbol{s}\}$ and $\breve{\boldsymbol{h}}\left(f_{\mathrm{D}}\right)$ and then present the networks with the best performance in the following.

We first consider the case that $\breve{h}\left(f_{\mathrm{D}}\right)$ is fed back to $\mathrm{BS}$. Obviously, the length of the vector $\widehat{\boldsymbol{h}}\left(f_{\mathrm{D}}\right)$ is $M-T_{\mathrm{fb}}$. As shown in Fig. 5] (a), we propose Net $\{\mathrm{P}\}$ to predict unknown $\widehat{\boldsymbol{h}}\left(f_{\mathrm{D}}\right)$ based on the known $\breve{\boldsymbol{h}}\left(f_{\mathrm{D}}\right)$, i.e., to learn the mapping $\boldsymbol{\Upsilon}$. The network structure of $\operatorname{Net}\{\mathrm{P}\}$ is the same with $\operatorname{Net}\{\mathrm{L}\}$ except that the input and the label of $\operatorname{Net}\{\mathrm{P}\}$ are $\boldsymbol{\xi} \circ \breve{\boldsymbol{h}}\left(f_{\mathrm{D}}\right)$ and $\boldsymbol{\xi} \circ \widehat{\boldsymbol{h}}\left(f_{\mathrm{D}}\right)$, respectively. As shown in Fig. 5 (b), we propose $\operatorname{Net}\{\mathrm{P}, \mathrm{H}\}$ to fuse the network output of $\operatorname{Net}\{\mathrm{P}\}$ and the hidden layer output of $\operatorname{Net}\{\mathrm{H}\}$. As presented in Fig. 5(c), we propose $\operatorname{Net}\{\mathrm{P}, \mathrm{H}, \mathrm{U}\}$ to fuse the network output of $\operatorname{Net}\{\mathrm{P}, \mathrm{H}\}$ and the hidden layer output of Net $\{\mathrm{U}\}$. As illustrated in Fig. 5(d), we propose $\operatorname{Net}\{\mathrm{P}, \mathrm{H}, \mathrm{U}, \mathrm{L}\}$ to fuse the network outputs of both $\operatorname{Net}\{\mathrm{H}, \mathrm{L}, \mathrm{U}\} \mathrm{h} 2$ and $\operatorname{Net}\{\mathrm{P}\}$.

Then, we consider the case that $r$ is fed back to BS. Since the length of the feedback vector $r$ is smaller than $M$, i.e., $T_{p}=T_{\mathrm{fb}}<M$, the LS estimator is not applicable due to the rank deficiency. However, it is feasible for DNNs to learn the mapping from $\{\boldsymbol{r}, \boldsymbol{s}\}$ to $\boldsymbol{h}\left(f_{\mathrm{D}}\right)$, and thus we propose $\operatorname{Net}\{\mathrm{R}\}$ to predict $\boldsymbol{h}\left(f_{\mathrm{D}}\right)$ base on $\{\boldsymbol{r}, \boldsymbol{s}\}$. As shown in Fig. 6 (a), $\boldsymbol{\xi} \circ \boldsymbol{r}$ is the input data of the first dense layer while $\Re[s]$ and $\Im[s]$ are concatenated at a new axis as the input data of the first convolution layer. Each convolution layer is followed by the LeakyReLU and the average pooling function. The average pooling functions are added to down-sample the data stream and avoid overfitting [53]. After reshaping the output of the last convolution layer, we use Net $\{$ Fus 0$\}$ to fuse the two data streams from the modalities $r$ and $s$. The label of $\operatorname{Net}\{R\}$ is $\boldsymbol{\xi} \circ \boldsymbol{h}\left(f_{\mathrm{D}}\right)$. Moreover, we propose $\operatorname{Net}\{\mathrm{R}, \mathrm{H}, \mathrm{U}\}$ to fuse the network output of $\operatorname{Net}\{R\}$ with the hidden layer outputs of both $\operatorname{Net}\{\mathrm{H}\}$ and $\operatorname{Net}\{\mathrm{U}\}$, as depicted in Fig. 6(b). We also propose $\operatorname{Net}\{R, H, L\}$ to fuse the hidden layer output of $\operatorname{Net}\{H\}$ with the network outputs of both $\operatorname{Net}\{R\}$ and Net $\{\mathrm{L}\}$, as shown in Fig. 6 (c). As illustrated in Fig. 6 (d), we propose $\operatorname{Net}\{R, H, L, U\}$ to fuse the network outputs of both $\operatorname{Net}\{\mathrm{H}, \mathrm{L}, \mathrm{U}\}_{\mathrm{h} 2}$ and $\operatorname{Net}\{\mathrm{R}\}$.

Remark 2: All the networks proposed in Section III-C can be easily extended to other problems like beam prediction and antenna selection. Specifically, by replacing the labels of all these networks $\boldsymbol{\xi} \circ \boldsymbol{h}\left(f_{\mathrm{D}}\right)$ with the optimal beam vectors, the proposed architectures can handle the beam prediction at the BS side. Besides, the proposed architectures can deal with antenna selection by replacing the labels of all these networks with the optimal selection vectors. It is worth mentioning that these variant architectures do not require prefect downlink channels, which can significantly reduce the cost resulted from downlink channel prediction. 


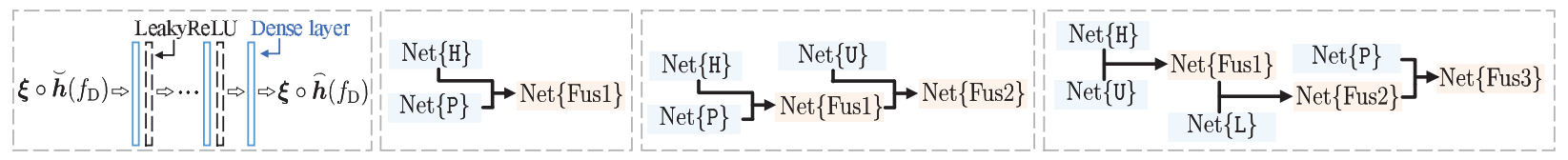

(a)

(b)

(c)

(d)

Fig. 5. The network structures of $\operatorname{Net}\{P\}$ (a), $\operatorname{Net}\{P, H\}(b), \operatorname{Net}\{P, H, U\}$ (c), and $\operatorname{Net}\{P, H, L, U\}$ (d).

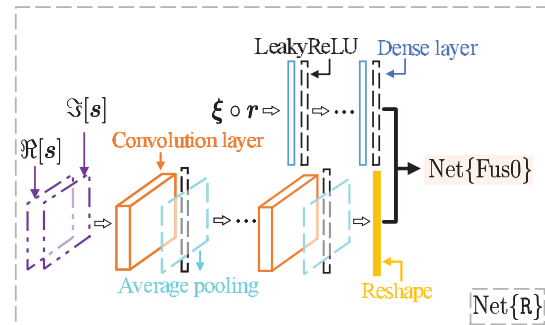

(a)

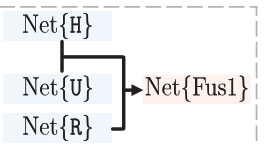

(b)

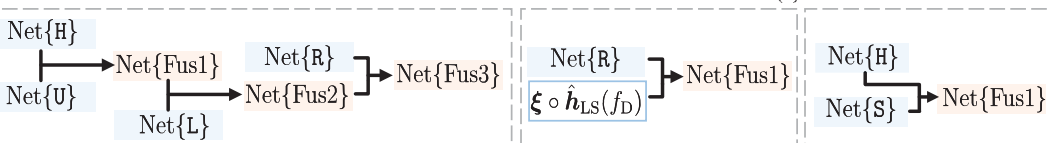

(d)

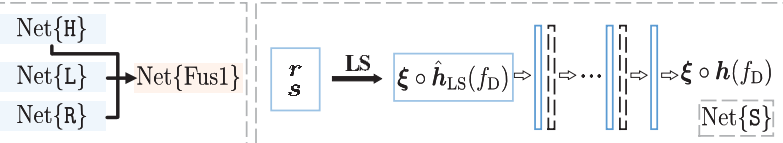

(c)

(f)

(g)

Fig. 6. The network structures of $\operatorname{Net}\{R\}$ (a), $\operatorname{Net}\{R, H, U\}(b), \operatorname{Net}\{R, H, L\}$ (c), Net $\{R, H, L, U\}(d), \operatorname{Net}\{S\}$ (e), Net $\{S, R\}(f)$, and $\operatorname{Net}\{S, H\}(g)$.

\section{Architecture Designs at the User Side}

We consider three different scenarios for downlink channel prediction at the user side, i.e., pilots being unavailable, insufficient or sufficient.

1) Pilots are unavailable: In this scenario, available modalities are the previous downlink channels $\overleftarrow{\boldsymbol{h}}\left(f_{\mathrm{D}}\right)$ and the user location $\boldsymbol{D}_{(x, y, z)}$. As described in Section III-C1, we can use $\operatorname{Net}\{H\}, \operatorname{Net}\{L\}, \operatorname{Net}\{H, L\}_{d}$ and $\operatorname{Net}\{H, L\}_{f}$ to predict the downlink channels.

2) Pilots are insufficient: In this scenario, available modalities are $\overleftarrow{\boldsymbol{h}}\left(f_{\mathrm{D}}\right), \boldsymbol{D}_{(x, y, z)},\{\boldsymbol{r}, \boldsymbol{s}\}$, and $\breve{\boldsymbol{h}}\left(f_{\mathrm{D}}\right)$. As described in Section III-C2 we can use $\operatorname{Net}\{R\}, \operatorname{Net}\{P, H\}$ and Net $\{R, H, L\}$ to predict the downlink channels.

3) Pilots are sufficient: When pilots are sufficient, the LS estimator can be used to estimate the downlink channel. Inspired by [8] and [10], we propose $\operatorname{Net}\{\mathrm{S}\}$, consisting of several dense layers and LeakyReLU functions, to predict $\boldsymbol{h}\left(f_{\mathrm{D}}\right)$ based on the LS estimate of downlink channel $\hat{\boldsymbol{h}}_{\mathrm{LS}}\left(f_{\mathrm{D}}\right)$, as illustrated in Fig. 6(e). The input and the label of Net $\{\mathrm{S}\}$ are $\boldsymbol{\xi} \circ \hat{\boldsymbol{h}}_{\mathrm{LS}}\left(f_{\mathrm{D}}\right)$ and $\boldsymbol{\xi} \circ \boldsymbol{h}\left(f_{\mathrm{D}}\right)$, respectively. It should be emphasized that even when LS estimates have been obtained, the available modalities, i.e., $\overleftarrow{\boldsymbol{h}}\left(f_{\mathrm{D}}\right)$ and $\{\boldsymbol{r}, \boldsymbol{s}\}$, could also be utilized to enhance the accuracy. Moreover, we propose the $\operatorname{Net}\{S, R\}$, as shown in Fig. 6 (f), where the network input of $\operatorname{Net}\{S\}$ and the network output of $\operatorname{Net}\{R\}$ are fused by Net\{Fus1\}. We also propose the $\operatorname{Net}\{\mathrm{S}, \mathrm{H}\}$ to fuse the network output of $\operatorname{Net}\{S\}$ and the hidden layer output of $\operatorname{Net}\{\mathrm{H}\}$, as displayed in Fig. 6(g).

Remark 3: The networks $\operatorname{Net}\{R\}, \operatorname{Net}\{R, H, L\}$, and $\operatorname{Net}\{S, R\}$ can be easily extended to data detection. One simple way inspired by [18] is to first divide the transmitted signals into pilots and data signals. Then, the pilots are fed to the network as depicted in Fig. 6 while the data signals are adopted as the training labels. In this way, we do not need to collect the downlink channels as training labels, which can significantly reduce the cost for label collection.

\section{E. Training Steps and Complexity Analyses}

The detailed training steps of all the proposed fusion networks are given as follow $\$$ :

1) Train the elementary networks, e.g., $\operatorname{Net}\{H\}, \operatorname{Net}\{L\}$, $\operatorname{Net}\{U\}, \operatorname{Net}\{P\}, \operatorname{Net}\{R\}$, and $\operatorname{Net}\{S\}$, independently to minimize the loss between its output and the label $\boldsymbol{\xi} \circ \boldsymbol{h}\left(f_{\mathrm{D}}\right)$ until their loss functions converge, and then fix these network parameters;

2) Train Net\{Fus1\} to minimize the loss between its output and the label $\boldsymbol{\xi} \circ \boldsymbol{h}\left(f_{\mathrm{D}}\right)$ until its loss function converges, and then fix its network parameters;

3) Following step 2), train Net $\{$ Fus 2$\}$ and Net $\{$ Fus 3$\}$ successively until their loss function converges and then fix their network parameters successively.

The required number of floating point operations is used as the metric of complexity. Then, the complexity of the dense layer is $O\left(n_{\text {in }} n_{\text {out }}\right)$, where $n_{\text {in }}$ and $n_{\text {out }}$ are respectively the input and the output data sizes. The complexity of the convolution layer is $O\left(n_{\text {in }} n_{\text {Flit }} n_{\text {Flit,in }} n_{\text {Flit,out }}\right)$, where $n_{\text {Flit }}$ is the filter size while $n_{\text {Flit,in }}$ and $n_{\text {Flit,out }}$ are respectively the input and the output numbers of filters. The complexity of the LSTM layer is $O\left(4 T_{\text {unit }} n_{\text {in }} n_{\text {out }}\right)$. Obviously, the computation cost increases as the number of modalities increases. The balance between the cost and the performance should be considered in practical applications.

\section{Simulation Results}

In this section, we will first present the simulation scenario and default network parameters. Then, the performance of the proposed networks will be evaluated and analyzed.

\footnotetext{
${ }^{6}$ The number of training iterations often increases as the network size increases. In order to accelerate the off-line training, we choose to train the elementary networks in advance and then fix these parameters. In fact, all the proposed fusion networks could also be trained end-to-end with its parameters initialized by the elementary networks.
} 


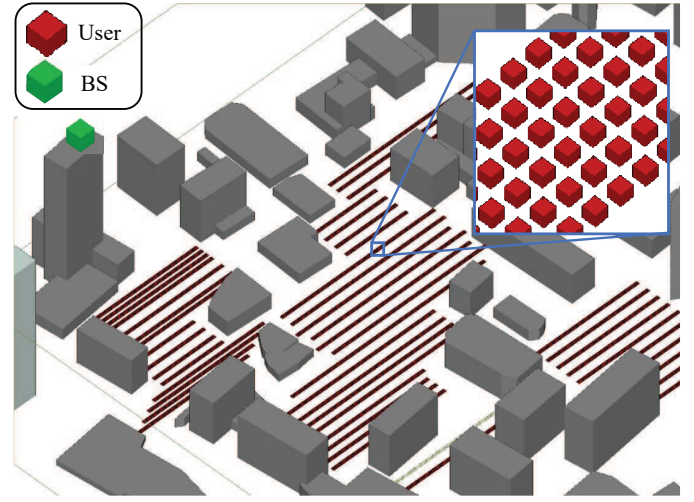

Fig. 7. A partial view of the ray-tracing scenario. The green little box represents the BS antennas. The red little box represents the possible location of the user antenna and the distance between adjacent red little boxes is $1 \mathrm{~m}$. The red line is consistent with the y-axis. This ray-tracing simulator shoots thousands of rays in all directions from the transmitter and records the strongest 25 paths that reach the receiver [46].

TABLE III

DEFAULT PARAMETERS FOR THE PROPOSED NETWORKS

\begin{tabular}{|c|c|c|c|}
\hline Modality & Network & Structure parameter & Learning rate \\
\hline$\overleftarrow{\boldsymbol{h}}\left(f_{\mathrm{D}}\right)$ & Net $\{\mathrm{H}\}$ & $T_{u}=3$, LSTM: 256,256 & $5 \mathrm{e}-4$ \\
\hline $\boldsymbol{D}_{(x, y, z)}$ & $\operatorname{Net}\{\mathrm{L}\}$ & Dense: $256,256,256,256$ & $1 \mathrm{e}-4$ \\
\hline $\boldsymbol{h}\left(f_{\mathrm{U}}\right)$ & $\operatorname{Net}\{\mathrm{U}\}$ & Dense: $256,256,256$ & $1 \mathrm{e}-3$ \\
\hline$\breve{\boldsymbol{h}}\left(f_{\mathrm{D}}\right)$ & $\operatorname{Net}\{\mathrm{P}\}$ & Dense: $256,256,256$ & $5 \mathrm{e}-4$ \\
\hline$\{\boldsymbol{r}, \boldsymbol{s}\}$ & $\operatorname{Net}\{\mathrm{R}\}$ & $\begin{array}{c}\text { Dense: } 256,128,128 \\
\text { Filter number: } 16,32,8 \\
\text { Filter: }(5,5)\end{array}$ & $5 \mathrm{e}-4$ \\
\hline$\hat{\boldsymbol{h}}_{\mathrm{LS}}\left(f_{\mathrm{D}}\right)$ & $\operatorname{Net}\{\mathrm{S}\}$ & Dense: 256 & $5 \mathrm{e}-4$ \\
\hline- & $\begin{array}{c}\text { Net }\{\mathrm{Fus} i\} \\
\mathrm{i}=0,1,2,3\end{array}$ & Dense: $512,512,256$ & $5 \mathrm{e}-4$ \\
\hline
\end{tabular}

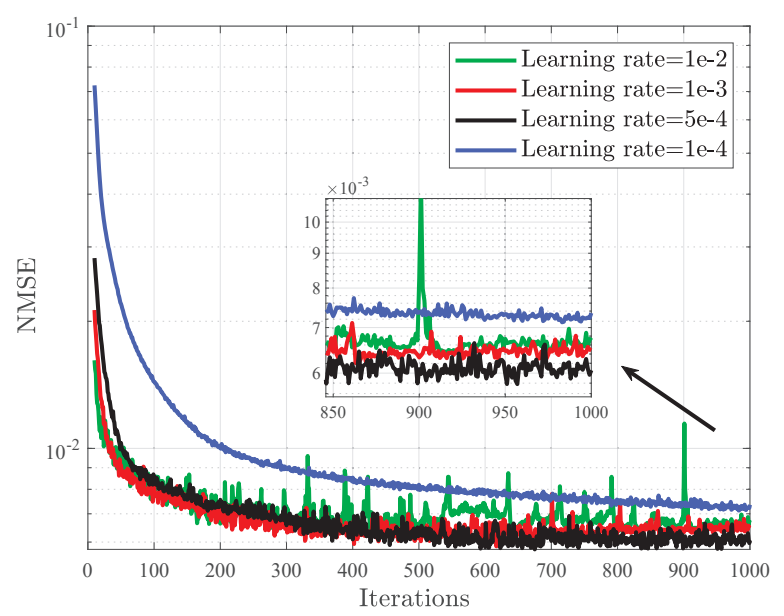

Fig. 8. The NMSE performance of $\operatorname{Net}\{\mathrm{H}\}$ versus different learning rates.

\section{A. Simulation Setup}

Dataset Generation: In the simulations, we consider the outdoor massive MIMO scenario that is constructed based on the accurate 3D ray-tracing simulator Wireless InSite [46]. Unlike conventional statistical channel generation methods, the $3 \mathrm{D}$ ray-tracing simulator can capture the dependence of channels on the environment geometry/materials and transmit- ter/receiver locations, and therefore can provide more reliable datasets for training and testing. The scenario comprises one BS and massive randomly distributed user antennas and each BS is equipped with 64 antennas. The scenario covers an area of $500 \times 500$ square metres. A partial view of the ray-tracing scenario is illustrated in Fig. 7 The uplink and downlink frequencies are set to be $2.50 \mathrm{GHz}$ and $2.62 \mathrm{GHz}$, respectively. Based on the environment setup, the $3 \mathrm{D}$ raytracing simulator first outputs the uplink channel parameters, the downlink channel parameters, and the location $\boldsymbol{D}_{(x, y, z)}$ for each user. Then, we can obtain $\boldsymbol{h}\left(f_{\mathrm{U}}\right)$ and $\boldsymbol{h}\left(f_{\mathrm{D}}\right)$ for each user by using Eq. (2) and the channel parameters from the 3D ray-tracing simulator. With Eq. (1), we can generate the pilots and the received signals $\{\boldsymbol{r}, \boldsymbol{s}\}$ based on $\boldsymbol{h}\left(f_{\mathrm{D}}\right)$. Assuming that the previous downlink channels are the channels of the user at adjacent positions, and the users move along the y-axis7. Then, $\overleftarrow{\boldsymbol{h}}\left(f_{\mathrm{D}}\right)$ can be obtained by collecting channels at adjacent $T_{\text {unit }}$ positions. Partial downlink channel $\breve{\boldsymbol{h}}\left(f_{\mathrm{D}}\right)$ can be obtained by selecting $M_{\mathrm{fb}}$ elements out of $\boldsymbol{h}\left(f_{\mathrm{D}}\right)$, and then the rest $M-M_{\mathrm{fb}}$ elements constitute the vector $\widehat{\boldsymbol{h}}\left(f_{\mathrm{D}}\right)$. After obtaining all sample pairs, we randomly select 9000 samples from the sample pairs as the training dataset, and select 1000 samples from the rest of sample pairs as the testing dataset. Since the perfect channels are not available in practical situation, unless otherwise specified, all the sample pairs in the datasets are estimated by the LMMSE algorithm [47] when the signal-tonoise ratio (SNR) is $25 \mathrm{~dB}$.

Adopted Neural Networks: Unless otherwise specified, the parameters of the proposed networks are given in Tab. III, where "LSTM: 256, 256" means that the hidden layers in Net $\{\mathrm{H}\}$ consist of two LSTM layers, and each hidden layer has 256 units. The numbers of units in the input and the output layers for all the proposed networks are consistent with the lengths of the input and the output data vectors, and thus are omitted in Tab. [III. We choose the output of the middle hidden layer as the hidden layer output of the networks. The batch size $V$ of all proposed networks is 128 . To facilitate the understanding, we also list the involved modalities for the elementary networks in Tab. III] For the proposed fusion networks, $\{\cdot\}_{\mathrm{f}},\{\cdot\}_{\mathrm{d}},\{\cdot\}_{\mathrm{h}}$ respectively represent the feature, the decision, and the hybrid fusions. For natation simplicity, some hybrid fusion networks have omitted the notation h, i.e., $\operatorname{Net}\{\mathrm{P}, \cdot\}, \operatorname{Net}\{\mathrm{R}, \cdot\}$, and $\operatorname{Net}\{\mathrm{S}, \cdot\}$.

Normalized mean-squared-error (NMSE) is used to measure the prediction accuracy, and is defined as

$$
\mathrm{NMSE}=E\left[\left\|\boldsymbol{h}_{\mathrm{D}}-\hat{\boldsymbol{h}}_{\mathrm{D}}\right\|_{2}^{2} /\left\|\boldsymbol{h}_{\mathrm{D}}\right\|_{2}^{2}\right],
$$

where $\hat{\boldsymbol{h}}_{D}$ and $\boldsymbol{h}_{D}$ represent the estimated and the true downlink channels, respectively. Note that all the parameters in Tab. III are basically selected by trails and errors such that these algorithms perform well. Take the learning rate selection of $\operatorname{Net}\{\mathrm{H}\}$ as an example. As illustrated in Fig. 8, the accuracy stability improves as the learning rate decreases. In particular,

\footnotetext{
${ }^{7}$ One similar example of using ray-tracing to model the time correlations can be found in [54]. For more details about how to generate channels using Wireless InSite, please refer to the paper [46] and the codes [55].
} 


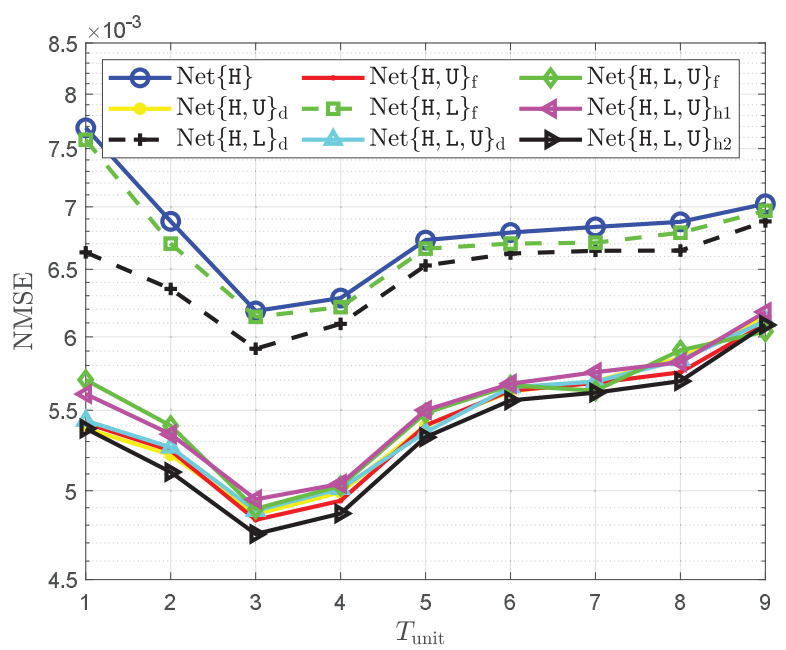

Fig. 9. The NMSE performance of previous downlink channel related networks versus $T_{\text {unit }}$

$\operatorname{Net}\{\mathrm{H}\}$ can achieve the highest accuracy when the learning rate is $5 \mathrm{e}-4$.

\section{B. BS Side}

Fig. 9 displays the NMSE performance of the previous downlink channel related networks versus $T_{\text {unit }}$. A larger $T_{\text {unit }}$ means that $\operatorname{Net}\{\mathrm{H}\}$ would learn time correlation of downlink channels from previous downlink channels in longer time periods. It can be observed that the performance of all these networks first improves and then degrades as $T_{\text {unit }}$ increases. This is because that the time correlations among the current channel and previous downlink channels can help to improve the performance of the proposed networks while the time correlations tend to vanish as $T_{\text {unit }}$ increases. Furthermore, $\operatorname{Net}\{\mathrm{H}\}$ consistently achieves the worst performance, and Net $\{\mathrm{H}, \mathrm{L}, \mathrm{U}\}_{\mathrm{h} 2}$ consistently outperforms other networks. This implies that the modalities $\boldsymbol{D}_{(x, y, z)}$ and $\boldsymbol{h}\left(f_{\mathrm{U}}\right)$ do provide complementary information for $\operatorname{Net}\{\mathrm{H}\}$. We will set $T_{\text {unit }}$ to be 3 for better performance in the following simulations.

Fig. 10 shows the NMSE performance of all networks that are applicable to BS without feedback link, as discussed in Section III-C1 As shown in Fig. 10, all the two-modalities fused networks outperform the corresponding two singlemodality networks, which implies that any two of the three modalities, i.e., $\overleftarrow{\boldsymbol{h}}\left(f_{\mathrm{D}}\right), \boldsymbol{D}_{(x, y, z)}$ and $\boldsymbol{h}\left(f_{\mathrm{U}}\right)$, can provide complementary information, thus enhancing the prediction accuracy. In particular, although $\operatorname{Net}\{\mathrm{L}\}$ has worse performance than both $\operatorname{Net}\{\mathrm{H}\}$ and $\operatorname{Net}\{\mathrm{U}\}$, the four two-modalities fused networks, i.e., $\operatorname{Net}\{\mathrm{H}, \mathrm{L}\}_{\mathrm{d}}, \operatorname{Net}\{\mathrm{H}, \mathrm{L}\}_{\mathrm{f}}, \operatorname{Net}\{\mathrm{L}, \mathrm{U}\}_{\mathrm{d}}$, and $\operatorname{Net}\{\mathrm{L}, \mathrm{U}\}_{\mathrm{f}}$, all have better performance than both $\operatorname{Net}\{\mathrm{H}\}$ and $\operatorname{Net}\{\mathrm{U}\}$. Besides, we notice that $\operatorname{Net}\{\mathrm{H}, \mathrm{U}\}_{\mathrm{f}}$ has the best performance among two-modalities fused networks, and the hybrid fusion network $\operatorname{Net}\{\mathrm{H}, \mathrm{L}, \mathrm{U}\}_{\mathrm{h} 2}$ outperforms other than feature or decision fusion networks. In fact, the structure of $\operatorname{Net}\{\mathrm{H}, \mathrm{L}, \mathrm{U}\}_{\mathrm{h} 2}$ is inspired by that of $\operatorname{Net}\{\mathrm{H}, \mathrm{U}\}_{\mathrm{f}}$. More specifically, since $\operatorname{Net}\{\mathrm{H}, \mathrm{U}\}_{\mathrm{f}}$ outperforms other two-modalities fused networks, we choose to preferentially fuse $\overleftarrow{\boldsymbol{h}}\left(f_{\mathrm{D}}\right)$ and $\boldsymbol{h}\left(f_{\mathrm{U}}\right)$ at the feature level. Following the $\operatorname{design}$ of $\operatorname{Net}\{\mathrm{H}, \mathrm{L}, \mathrm{U}\}_{\mathrm{h} 2}$, we develop the network structures of other fusion networks and only present these with best performance, i.e., $\operatorname{Net}\{\mathrm{P}, \cdot\}$, $\operatorname{Net}\{R, \cdot\}$, and $\operatorname{Net}\{S, \cdot\}$, which are exactly all hybrid fusion network. In summary, compared with the feature and the decision fusion networks, hybrid fusion networks can often achieve better performance but require higher design complexity. Moreover, the superiority of the feature and the decision fusion depends on the specific task.

Fig. 11 compares the NMSE performance of all the networks that are applicable to BS with feedback link, as discussed in Section III-C2 As shown in Fig. 11, the performance of all the proposed networks improves when the feedback length $T_{\mathrm{fb}}$ increases. When $T_{\mathrm{fb}}$ is small, the received signal based networks (solid lines) outperform partial downlink channel based networks (dotted lines). This is because the correlations between the received signals and downlink channels are stronger than the correlations between the channels of different antennas. Since the estimate error of the unknown channels becomes insignificant as $T_{\mathrm{fb}}$ increases, the prediction accuracy of partial downlink channel based networks would outperform the received signal based networks. Consider a extreme case, where $T_{\mathrm{fb}}$ equals 64 . Then, the NMSE of partial downlink channel based networks would be 0 . Furthermore, it can be observed from the second enlarge picture that $\operatorname{Net}\{\mathrm{P}, \mathrm{H}\}$ and $\operatorname{Net}\{P, H, L, U\}$ consistently outperform $\operatorname{Net}\{P\}$ while the gaps between the three networks all degrade as $T_{\mathrm{fb}}$ increases. This indicates that when we choose to feed partial downlink channel back, i.e., $T_{\mathrm{fb}} \geq 48$, we can adopt $\operatorname{Net}\{\mathrm{P}\}$ instead of other $\breve{h}\left(f_{\mathrm{D}}\right)$ related fusion networks to reduce the training cost, since the gaps between them are negligible. Moreover, as shown in the third enlarge picture, $\operatorname{Net}\{R, H, L, U\}$ consistently outperforms $\operatorname{Net}\{R\}$, Net $\{R, H, U\}$, and $\operatorname{Net}\{R, H, L\}$ while the gap between $\operatorname{Net}\{R, H, L, U\}$ and $\operatorname{Net}\{R\}$ becomes negligible when $T_{\mathrm{fb}}$ is larger than 36 . This indicates that we can adopt $\operatorname{Net}\{R, H, L, U\}$ for better prediction accuracy when $T_{\mathrm{fb}}$ is smaller than 36 and adopt $\operatorname{Net}\{R\}$ for lower training cost when $T_{\mathrm{fb}}$ is greater than 36 and less than 48 . Besides, when the feedback length is low, the extra accuracy gain obtained by using multi-modal data is remarkable, which also demonstrates the significant superiority of DML in FDD massive MIMO systems.

\section{User Side}

Fig. 12 displays the NMSE performance of LS, LMMSE, $\operatorname{Net}\{R\}, \operatorname{Net}\{S\}, \operatorname{Net}\{S, R\}$, and $\operatorname{Net}\{S, H\}$ versus the pilot length $T_{p}$, where SNR is $10 \mathrm{~dB}$ in both the training and the testing stages. The pervious channels involved in $\operatorname{Net}\{\mathrm{S}, \mathrm{H}\}$ are estimated by the LMMSE algorithm when SNR is 10 dB. As shown in Fig. 12, both $\operatorname{Net}\{\mathrm{S}, \mathrm{H}\}$ and $\operatorname{Net}\{\mathrm{S}, \mathrm{R}\}$ outperform $\operatorname{Net}\{S\}$ and $\operatorname{Net}\{R\}$, which means that extra modalities can provide complementary information to improve the prediction accuracy. Furthermore, Net $\{\mathrm{S}, \mathrm{H}\}$ outperforms all other algorithms, including the LMMSE algorithm, which demonstrates its remarkable effectiveness. 


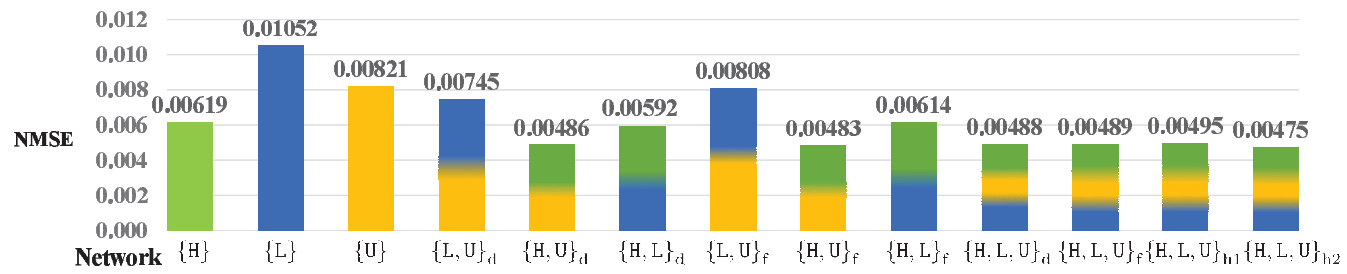

Fig. 10. The NMSE performance of the networks that are applicable to BS without feedback link.

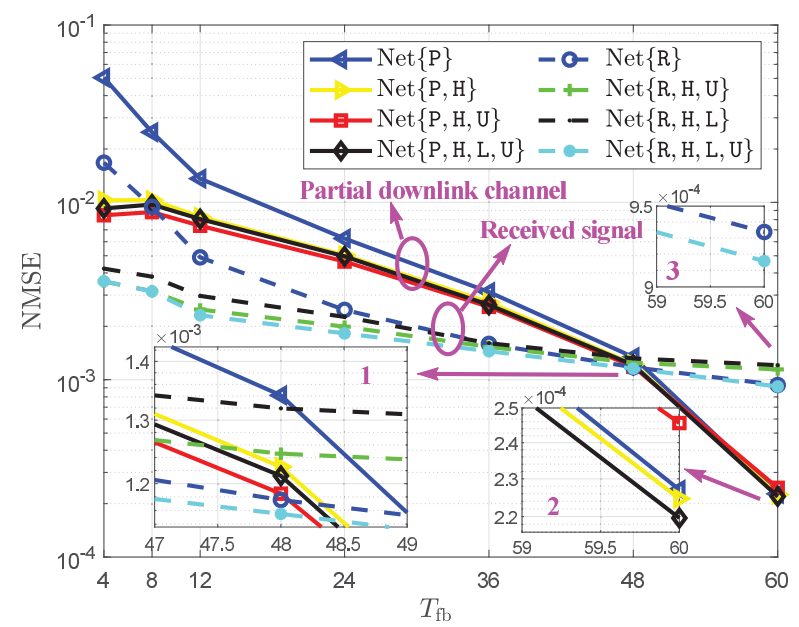

Fig. 11. The NMSE performance of all the networks that are applicable to BS with feedback link. The networks are trained separately for each value of $T_{\mathrm{bf}}$.

\section{Impairments in Practical Applications}

To collect off-line training samples, we can obtain extremely accurate channels by increasing SNR and the pilot length. However, in the on-line testing stage, varying SNRs would impair the prediction accuracy of the proposed networks. Therefore, we investigate the impact of various SNRs on the performance of LS, LMMSE, $\operatorname{Net}\{R\}, \operatorname{Net}\{S\}, \operatorname{Net}\{S, R\}$, $\operatorname{Net}\{\mathrm{S}, \mathrm{H}\}, \operatorname{Net}\{\mathrm{H}\}, \operatorname{Net}\{\mathrm{U}\}$, and $\operatorname{Net}\{\mathrm{H}, \mathrm{L}, \mathrm{U}\} \mathrm{h}_{\mathrm{h} 2}$, where $T_{p}$ is 64. Fig. 13 shows the performance of these networks versus the SNR in the on-line testing stage, where the networks are trained at $\mathrm{SNR}=10 \mathrm{~dB}$ and tested separately for each value of SNR. As indicated in Fig. 13, Net $\{\mathrm{S}, \mathrm{H}\}$ outperforms all other algorithms when SNR is lower than $15 \mathrm{~dB}$. This is because that the estimation based on the pilots and the received signals relies on high SNRs while the prediction based on pervious downlink channels is more robust over low SNRs. Since the pilots and the received signals would be more correlated with the downlink channel as the SNR increases, pilot related networks would outperform $\operatorname{Net}\{\mathrm{H}\}, \operatorname{Net}\{\mathrm{U}\}$, and Net $\{\mathrm{H}, \mathrm{L}, \mathrm{U}\}_{\mathrm{h} 2}$ in high SNR region. Moreover, the remarkable robustness of $\operatorname{Net}\{\mathrm{S}, \mathrm{H}\}$ over low SNRs also demonstrates its potential in real applications.

Discussion: The design and the testing for DML are interoperable, which is a typical characteristic of data-driven techniques. On the contrary, conventional communications are model-driven, where the algorithm designs rely on prerequisite models and their performance is completely predictable and explicable. In this paper, we have provided a heuristic frame-

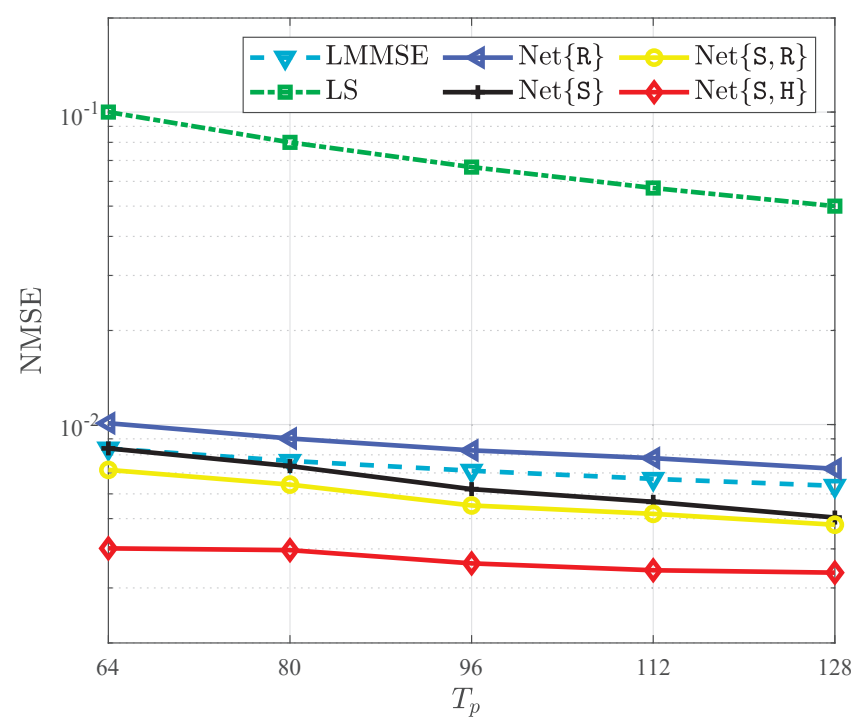

Fig. 12. The NMSE performance of the proposed networks versus the pilot length $T_{p}$. The networks are trained separately for each value of $T_{p}$, and SNR is $10 \mathrm{~dB}$ in both the training and the testing stages.

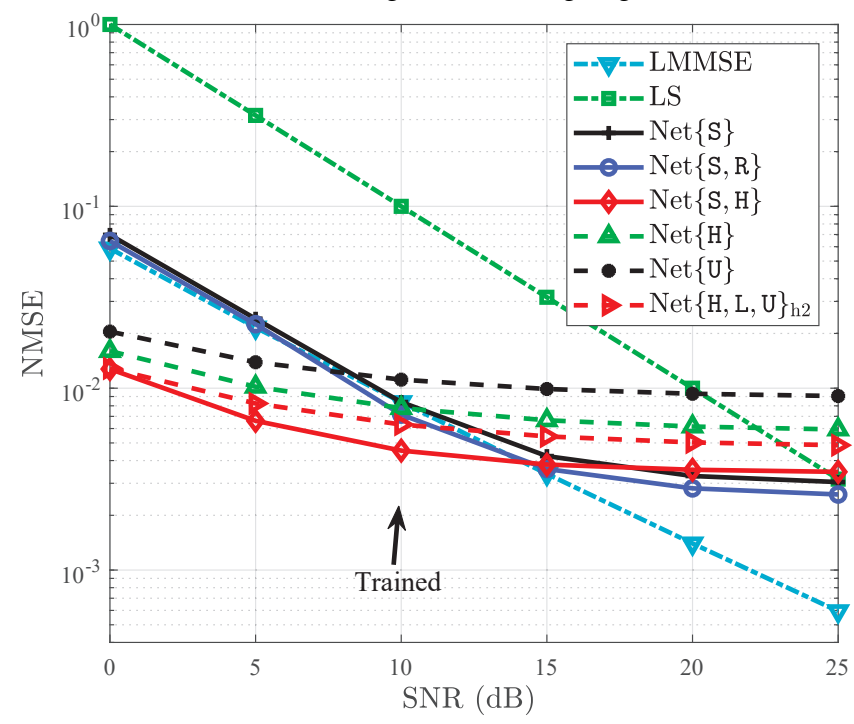

Fig. 13. The NMSE performance of the proposed networks versus SNR, where $T_{p}$ is 64 . The networks are trained at $\mathrm{SNR}=10 \mathrm{~dB}$ and tested separately for each value of SNR.

work for DML based communications, and the theoretical guidance on the framework will be left for future work. It should be mentioned that the proposed DML based framework can also be used in cell-free or multi-cell massive MIMO systems [2], [16]. 


\section{CONCLUSION}

In this paper, we introduced DML into wireless communications to fully exploit the MSI in communication systems such that the system performance could be improved. We provided complete descriptions and heuristic analyses of the design choices in DML based wireless communications. We also proposed several efficient DML based architectures for channel prediction as a case study. Simulation results have shown that DML based architectures exhibit significant advantages over one modality based architectures under most cases, which also demonstrate that the proposed framework can effectively exploit the constructive and complementary information of multimodal sensory data to assist the current wireless communications.

\section{REFERENCES}

[1] T. Wang, C. Wen, H. Wang, F. Gao, T. Jiang, and S. Jin, "Deep learning for wireless physical layer: Opportunities and challenges," China Commun., vol. 14, no. 11, pp. 92-111, Nov. 2017.

[2] J. Zhang, E. Bjórnson, M. Matthaiou, D. W. K. Ng, H. Yang, and D. J. Love, "Prospective multiple antenna technologies for beyond 5G," IEEE J. Selected Areas Commun., vol. 38, no. 8, pp. 1637-1660, Jun. 2020.

[3] H. He, S. Jin, C. Wen, F. Gao, G. Y. Li, and Z. Xu, "Model-driven deep learning for physical layer communications," IEEE Wireless Commun., vol. 26, no. 5, pp. 77-83, Oct. 2019.

[4] Z. Qin, H. Ye, G. Y. Li, and B. F. Juang, "Deep learning in physical layer communications," IEEE Wireless Commun., vol. 26, no. 2, pp. 93-99, Apr. 2019.

[5] Y. Yang, F. Gao, C. Qian, and G. Liao, "Model-aided deep neural network for source number detection," IEEE Signal Process. Lett., vol. 27, pp. 91-95, 2020.

[6] H. He, C. Wen, S. Jin, and G. Y. Li, "Deep learning-based channel estimation for beamspace mmwave massive MIMO systems," IEEE Wireless Commun. Lett., vol. 7, no. 5, pp. 852-855, Oct. 2018.

[7] P. Dong, H. Zhang, G. Y. Li, I. S. Gaspar, and N. NaderiAlizadeh, "Deep CNN-based channel estimation for mmwave massive MIMO systems," IEEE J. Sel. Topics Signal Process., vol. 13, no. 5, pp. 989-1000, Sept. 2019.

[8] Y. Yang, F. Gao, X. Ma, and S. Zhang, "Deep learning-based channel estimation for doubly selective fading channels," IEEE Access, vol. 7, pp. 36579-36589, Mar. 2019

[9] X. Ma, H. Ye, and Y. Li, "Learning assisted estimation for time-varying channels," in Proc. 15th Int. Symp. Wireless Commun. Systems (ISWCS), Lisbon, Portugal, Aug. 2018, pp. 1-5.

[10] X. Gao, S. Jin, C. Wen, and G. Y. Li, "Comnet: Combination of deep learning and expert knowledge in OFDM receivers," IEEE Commun. Lett., vol. 22, no. 12, pp. 2627-2630, Oct. 2018.

[11] J. Yuan, H. Q. Ngo, and M. Matthaiou, "Machine learning-based channel prediction in massive MIMO with channel aging," IEEE Trans. Wireless Commun., vol. 19, no. 5, pp. 2960-2973, Feb. 2020.

[12] Y. Yang, F. Gao, Z. Zhong, B. Ai, and A. Alkhateeb, "Deep transfer learning based downlink channel prediction for FDD massive MIMO systems," IEEE Trans. Commun., pp. 1-1, 2020.

[13] M. Alrabeiah and A. Alkhateeb, "Deep learning for TDD and FDD massive MIMO: Mapping channels in space and frequency," in Proc. 53rd Asilomar Conf. Signals, Systems, Computers, California, USA, 2019, pp. 1465-1470.

[14] Y. Yang, F. Gao, G. Y. Li, and M. Jian, "Deep learning based downlink channel prediction for FDD massive MIMO system," IEEE Commun. Lett., vol. 23, no. 11, pp. 1994-1998, Nov. 2019.

[15] Y. Jin, J. Zhang, B. Ai, and X. Zhang, "Channel estimation for mmwave massive MIMO with convolutional blind denoising network," IEEE Commun. Lett., vol. 24, no. 1, pp. 95-98, Aug. 2020.

[16] Y. Jin, J. Zhang, S. Jin, and B. Ai, "Channel estimation for cell-free mmwave massive MIMO through deep learning," IEEE Trans. Vehicular Technol., vol. 68, no. 10, pp. 10325-10329, Nov. 2019.

[17] P. Dong, H. Zhang, and G. Y. Li, "Machine learning prediction based CSI acquisition for FDD massive MIMO downlink," in Proc. IEEE Global Commun. Conf. (GLOBECOM), Abu Dhabi, United Arab Emirates, Dec. 2018, pp. 1-6.
[18] H. Ye, G. Y. Li, and B. Juang, "Power of deep learning for channel estimation and signal detection in OFDM systems," IEEE Wireless Commun. Lett., vol. 7, no. 1, pp. 114-117, Feb. 2018.

[19] H. He, C. Wen, S. Jin, and G. Y. Li, "Model-driven deep learning for MIMO detection," IEEE Trans. Signal Process., vol. 68, pp. 1702-1715, Feb. 2020.

[20] T. Wang, C. Wen, S. Jin, and G. Y. Li, "Deep learning-based CSI feedback approach for time-varying massive MIMO channels," IEEE Wireless Commun. Lett., vol. 8, no. 2, pp. 416-419, Apr. 2019.

[21] J. Guo, C. Wen, S. Jin, and G. Y. Li, "Convolutional neural network based multiple-rate compressive sensing for massive MIMO CSI feedback: Design, simulation, and analysis," IEEE Trans. Wireless Commun., vol. 19, no. 4, pp. 2827-2840, Apr. 2020.

[22] M. Alrabeiah and A. Alkhateeb, "Deep learning for mmwave beam and blockage prediction using Sub-6GHz channels," IEEE Trans. Commun., vol. 68 , no. 9 , pp. 5504-5518, Jun. 2020.

[23] W. Xu, F. Gao, S. Jin, and A. Alkhateeb, "3D scene based beam selection for mmwave communications," IEEE Wireless Commun. Lett., pp. 1-1, 2020.

[24] M. Alrabeiah, A. Hredzak, Z. Liu, and A. Alkhateeb, "ViWi: a deep learning dataset framework for vision-aided wireless communications," in Proc. IEEE 91st Vehicular Technol. Conf. (VTC), Antwerp, Belgium, 2020, pp. $1-5$.

[25] X. Li and A. Alkhateeb, "Deep learning for direct hybrid precoding in millimeter wave massive MIMO systems," in 53rd Asilomar Conf. Signals, Systems, Computers, California, USA, Nov. 2019, pp. 800-805.

[26] B. Wang, F. Gao, S. Jin, H. Lin, and G. Y. Li, "Spatial- and frequencywideband effects in millimeter-wave massive MIMO systems," IEEE Trans. Signal Process., vol. 66, no. 13, pp. 3393-3406, Jul. 2018.

[27] T. L. Marzetta, "Noncooperative cellular wireless with unlimited numbers of base station antennas," IEEE Tran. Wireless Commun., vol. 9, no. 11, pp. 3590-3600, Oct. 2010.

[28] P. Liu, S. Jin, T. Jiang, Q. Zhang, and M. Matthaiou, "Pilot power allocation through user grouping in multi-cell massive MIMO systems," IEEE Trans. Commun., vol. 65, no. 4, pp. 1561-1574, Dec. 2017.

[29] E. Bjórnson, J. Hoydis, and L. Sanguinetti, "Massive MIMO has unlimited capacity," IEEE Trans. Wireless Commun., vol. 17, no. 1, pp. 574-590, Nov. 2018.

[30] D. Ramachandram and G. W. Taylor, "Deep multimodal learning: A survey on recent advances and trends," IEEE Signal Process. Mag., vol. 34, no. 6, pp. 96-108, 2017.

[31] J. Ngiam, A. Khosla, M. Kim, J. Nam, H. Lee, and A. Y. Ng, "Multimodal deep learning," in Proc. 28th Int. Conf. Int. Conf. Machine Learning (ICML), Madison, WI, USA, Jun. 2011, pp. 689696, Omnipress.

[32] C. Feichtenhofer, A. Pinz, and A. Zisserman, "Convolutional two-stream network fusion for video action recognition," in Proc. IEEE Conf. Computer Vision Pattern Recognition (CVPR), Las Vegas, USA, Jun. 2016, pp. 1933-1941.

[33] N. Srivastava and R. Salakhutdinov, "Learning representations for multimodal data with deep belief nets," in Proc. Int. conf. machine learning workshop, 2012, vol. 79.

[34] G. Hinton, L. Deng, D. Yu, G. E. Dahl, A. Mohamed, N. Jaitly, A. Senior, V. Vanhoucke, P. Nguyen, T. N. Sainath, and B. Kingsbury, "Deep neural networks for acoustic modeling in speech recognition: The shared views of four research groups," IEEE Signal Process. Mag., vol. 29, no. 6, pp. 82-97, Oct. 2012.

[35] P. Atrey, M. Hossain, A. El Saddik, and M. Kankanhalli, "Multimodal fusion for multimedia analysis: A survey," Multimedia Syst., vol. 16, no. 6 , pp. $345-379$, Nov. 2010.

[36] F. Zhu, F. Gao, H. Lin, S. Jin, J. Zhao, and G. Qian, "Robust beamforming for physical layer security in BDMA massive MIMO," IEEE J. Selected Areas Commun., vol. 36, no. 4, pp. 775-787, Apr. 2018.

[37] G. Potamianos, C. Neti, G. Gravier, A. Garg, and A. W. Senior, "Recent advances in the automatic recognition of audiovisual speech," Proc. IEEE, vol. 91, no. 9, pp. 1306-1326, Sept. 2003.

[38] D. Tse and P. Viswanath, Fundamentals of wireless communication, Cambridge university press, 2005.

[39] Y. Wu, E. Chang, K. Chang, and J. Smith, "Optimal multimodal fusion for multimedia data analysis," in Proc. 12th Annual ACM Int. Conf. Multimedia, New York, NY, USA, 2004, pp. 572-579, Association for Computing Machinery.

[40] P. Atrey, M. Kankanhalli, and J. Oommen, "Goal-oriented optimal subset selection of correlated multimedia streams," ACM Trans. Multimedia Comput. Commun. Appl., vol. 3, no. 1, pp. 2, Feb. 2007. 
[41] S. Lemmelä, "Selecting optimal modalities for multimodal interaction in mobile and pervasive environments," in Proc. Improved Mobile User Experience workshop, May 2008.

[42] R. Bellman, "Dynamic programming," 1957.

[43] M. Belkin and P. Niyogi, "Laplacian eigenmaps for dimensionality reduction and data representation," Neural Computation, vol. 15, no. 6, pp. 1373-1396, 2003.

[44] J. Kittler, M. Hatef, R. P. W. Duin, and J. Matas, "On combining classifiers," IEEE Trans. Pattern Analysis Machine Intelligence, vol. 20, no. 3, pp. 226-239, Mar. 1998.

[45] H. Xie, F. Gao, S. Zhang, and S. Jin, "A unified transmission strategy for TDD/FDD massive MIMO systems with spatial basis expansion model," IEEE Trans. Vehicular Technol., vol. 66, no. 4, pp. 3170-3184, Apr. 2017.

[46] A. Alkhateeb, "DeepMIMO: A generic deep learning dataset for millimeter wave and massive MIMO applications," in Proc. Inf. Theory and Applications Workshop (ITA), San Diego, CA, Feb. 2019, pp. 1-8.

[47] M. Biguesh and A. B. Gershman, "Training-based MIMO channel estimation: a study of estimator tradeoffs and optimal training signals," IEEE Trans. Signal Process., vol. 54, no. 3, pp. 884-893, Mar. 2006.

[48] J. Vieira, E. Leitinger, M. Sarajlic, X. Li, and F. Tufvesson, "Deep convolutional neural networks for massive MIMO fingerprint-based positioning," in Proc. IEEE 28th Annual Int. Symposium Personal, Indoor, Mobile Radio Communications (PIMRC), Montreal, Canada, Oct. 2017, pp. 1-6.

[49] K. Hornik, M. Stinchcombe, and H. White, "Multilayer feedforward networks are universal approximators," Neural netw., vol. 2, no. 5, pp. 359-366, 1989.

[50] H. Xie, F. Gao, S. Jin, J. Fang, and Y. Liang, "Channel estimation for TDD/FDD massive MIMO systems with channel covariance computing," IEEE Trans. Wireless Commun., vol. 17, no. 6, pp. 4206-4218, Jun. 2018.

[51] A. Maas, A. Hannun, and A. Ng, "Rectifier nonlinearities improve neural network acoustic models," in Proc. 30th Int. Conf. Machine Learning (ICML), Atlanta, Georgia, USA, 2013, vol. 30, p. 3.

[52] D. Kingma and J. Ba, "Adam: A method for stochastic optimization," arXiv preprint arXiv:1412.6980, 2014.

[53] M. Sun, Z. Song, X. Jiang, J. Pan, and Y. Pang, "Learning pooling for convolutional neural network," Neurocomputing, vol. 224, pp. 96-104, Feb. 2017.

[54] M. M. Taygur and T. F. Eibert, "Analyzing the channel aging effects on massive MIMO downlink by ray-tracing," in Proc. IEEE 29th Annual Int. Symposium Personal, Indoor Mobile Radio Commun.(PIMRC), Bologna, Italy, 2018, pp. 1-5.

[55] "Codes," [Online]. Available: https://github.com/yangyuwenyang/Codes-for-DML 